\title{
A survey of lifespans in Oribatida excluding Astigmata (Acari)
}

\author{
TOBIAS PFINGSTL ${ }^{1, *} \&$ HEINRICH SCHATZ ${ }^{2}$ \\ ${ }^{1}$ Institute of Biology, University of Graz, Universitätsplatz 2, $8010 \mathrm{Graz}$, Austria. \\ ”tobias.pfingstl@uni-graz.at; • \ttps://orcid.org/0000-0002-0778-8051 \\ ${ }^{2}$ clo Institute of Zoology, University of Innsbruck, Technikerstr. 25, 6020 Innsbruck, Austria. \\ ”"heinrich.schatz@uibk.ac.at; @ https://orcid.org/0000-0002-1338-3579 \\ ${ }^{*}$ Corresponding author
}

\begin{abstract}
This contribution provides an update on the duration of life cycles and lifespans of oribatid mites based on a literature review. The total lifespan is the sum of the immature developmental time (egg to adult) and the longevity of the adult. Most investigations were carried out in the laboratory, few were performed in the field, under field conditions and/or compared with field data. Many life cycles were investigated under different environmental influences. The life cycles of 144 oribatid species are listed. Compared with the total number of known oribatid species, this number is very low. Data for the total lifespan are given for 52 species, either from observations in the laboratory or estimated in comparison with field studies, but can only be guesses of the real lifespan. The typical lifespan of an oribatid species in temperate or boreal regions lasts between 1 and 2 years, rarely 3 years. The few investigated tropical species from laboratory experiments show generally faster development and shorter lifespans as species from temperate regions; no field studies have been carried out in the tropics yet. Long lifespan periods of 5 to 8 years are particularly characteristic of species in polar regions and in mountainous temperate regions. Some examples of species with different longevity in distinct climate regions, very long lifespans and change of life parameters under stressful laboratory conditions are presented.
\end{abstract}

Keywords: life-history, life cycle, development, longevity, generation time

\section{Introduction}

The oribatid mites (Acari, Oribatida) are a large group of minute free-living organisms, mainly terrestrial in organic soil litter, in moss or on trees, some species are aquatic (Behan-Pelletier \& Eamer 2007; Schatz \& Behan-Pelletier 2008), and some occur in the marine littoral zone (Pfingstl 2017). At present there are about 11.500 species known (Subías 2004, unpublished update 2021). The Astigmata, which have phylogenetic relationships to Oribatida (Norton 1998), have evolved different morphological and biological characters and largely a different mode of life, and are not considered here.

The ontogenetic development of Oribatida shows the complete sequence of the Chelicerata with six postembryonic instars: an immovable and inactive prelarva, a hexapod mobile larva, three octopod nymphal stages (proto-, deuto-, tritonymph, all morphologically differentiated by further development), and the reproductive adult stage (Walter \& Proctor 2013). Variations and exceptions from this scheme are known in some larviparous taxa, mainly in aquatic and marine littoral environment (Norton 1994; Behan-Pelletier \& Eamer 2007). All instars from larva to adult are separated by moulting. It should be noted that Oribatida have an unusually high rate of parthenogenesis. Almost 10\% of the known species may be thelytokous, most concentrated in a few families (Norton \& Palmer 1991; Norton et al. 1993). Generally, Oribatida are "Kstrategists" with low metabolic rate, low fecundity and reproductive output, and comparatively longer life cycles (Mitchell 1977a; Norton 1994; Norton \& Behan-Pelletier 2009). Most species are iteroparous with relatively long-living adults (Norton et al. 1993). Few exceptions from this scheme are known from species in extreme or unpredictable habitats (such as hot deserts, fresh water), with short developmental times and high reproduction rates characteristic for opportunistic "R-strategists" in unstable ecosystems (Wallwork 1980; Fernandez \& Athias Binche 1986; Wallwork et al. 1986). 
The total lifespan of an oribatid mite is the sum of the immature developmental time and the possible longevity of the adult. It is therefore essential for the estimation of total lifespan to consider the development from egg to adult. The term "life cycle" is mostly used as development from egg of the first generation to the egg of the second generation (generation time). Norton and Ermilov (2014, unpublished update 2021) list almost 900 oribatid species with morphological descriptions of juvenile instars. C.L. Koch described and named some immature oribatid mites (e.g., Koch $1835 ; 1839 ; 1841)$ although he was not aware that these were juvenile instars. Nicolet (1855) was the first author who studied the development of oribatid species in the laboratory, followed by Michael (1880) who also recognized the "potential for juveniles to inform classification" (a detailed history of studies on immature oribatid mites is given in Norton \& Ermilov 2014).

Michael (1884) observed the developmental time of some oribatid species in the laboratory. Since then, the duration of development from egg to adult has been published frequently. Most investigations were carried out in culture under simple laboratory conditions at room temperature (e.g., Michael 1884; Grandjean 1950), some combined with feeding experiments (e.g., Denmark \& Woodring 1965; Seniczak et al. 2016), or under controlled constant or variable temperature regimes (e.g., Woodring \& Cook 1962; Weigmann 1975; Bhattacharya et al. 1978), or under seminatural conditions which approximated field conditions (in field enclosures, e.g., Harding 1971, Lebrun 1971), some were also compared with the phenology from the field data (e.g., Block 1965; Lebrun 1970b; Weigmann 1975; Bellido 1979; Luxton 1981b; West 1982). Older data on developmental rates have been summarized by Lebrun (1970a — 36 species), Luxton (1981a—51 spp.), Kaneko (1988b - 43 spp.) and Grishina (1991-75 spp.). In the recent decades the studies of life cycles declined and concentrated on a few species (see results and Table 1). The causes of the differing lengths of life cycles such as species-specific, ecological or phylogenetic aspects have been discussed by several authors (e.g., Mitchell 1977a; Luxton 1981a; Norton et al. 1993; Norton 1994; Siepel 1994; Lebrun et al. 1991; Lebrun \& van Straalen 1995; Bale et al. 1997; Belozerov 2008). Norton (1994) outlined the resulting consequences for survivorship and mortality from the available data from field and laboratory observations.

In contrast to the frequently investigated life cycles, the total lifespan of Oribatida has been observed or calculated much less frequently. Luxton (1981a) provided a list of the longevity in the laboratory for 13 species of adult oribatid mites, based on the results of different authors. This contribution, as a literature review, summarizes the present stage of studies on lifespan of oribatid mites.

TABLE 1. Lifespan data (in days) for oribatid mites observed in laboratory or in field studies. 'Longevity' refers only to the duration of adult stage, 'lifespan' gives the full duration of life including juvenile development. 'Egg-adult' is the time from egg deposition to hatching of the adult (mostly referred to as developmental time or life-cycle) and 'egg-egg' is the time from egg deposition of the first generation to egg deposition of the second generation (also frequently referred to as generation time).

\begin{tabular}{|c|c|c|c|c|c|c|}
\hline Taxon & Longevity & Lifespan & Egg-adult & Egg-egg & Rearing & References and notes \\
\hline \multicolumn{7}{|l|}{ Palaeacaridae } \\
\hline Palaeacarus kamenskii & - & 240 & 91 & - & $\mathrm{lab} / 25^{\circ} \mathrm{C}$ & Shereef (1972) \\
\hline \multicolumn{7}{|l|}{ Brachychthoniidae } \\
\hline $\begin{array}{l}\text { Eobrachychthonius } \\
\text { oudemansi }\end{array}$ & - & - & 730 & - & $\begin{array}{l}\text { field / subantarctic } \\
\text { climate }\end{array}$ & West (1982) \\
\hline \multicolumn{7}{|l|}{ Hypochthoniidae } \\
\hline Eohypochthonius magnus & - & - & $420-450$ & 730 & field & Kaneko (1989), Japan \\
\hline \multirow[t]{3}{*}{ Hypochthonius rufulus } & - & - & - & 730 & field & Luxton (1981a), Denmark \\
\hline & - & - & 122 & - & $\mathrm{lab} / 25^{\circ} \mathrm{C}$ & Sengbusch (1958b) \\
\hline & $<2$ & & & & $\mathrm{lab} / 5-25^{\circ} \mathrm{C}$ & $\begin{array}{l}\text { Madge (1964), } \\
\text { reaction to temperature and } \\
\text { relative humidity }\end{array}$ \\
\hline \multicolumn{7}{|l|}{ Lohmanniidae } \\
\hline Lepidacarus ornatissimus & - & - & 178 & - & lab $/ 30 \pm 1^{\circ} \mathrm{C}$ & Haq \& Adolph (1981) \\
\hline Papillacarus aciculatus & 730 & - & 71 & 95 & $\mathrm{lab} / 25^{\circ} \mathrm{C}$ & Shereef (1976b) \\
\hline
\end{tabular}


TABLE 1. (Continued)

\begin{tabular}{|c|c|c|c|c|c|c|}
\hline Taxon & Longevity & Lifespan & Egg-adult & Egg-egg & Rearing & References and notes \\
\hline Papillacarus egypticus & 730 & & 101 & 116 & $\mathrm{lab} / 25^{\circ} \mathrm{C}$ & $\begin{array}{l}\text { Shereef (1976b), } \\
\text { sub Lohmannia egypticus }\end{array}$ \\
\hline \multicolumn{7}{|l|}{ Mesoplophoridae } \\
\hline Archoplophora rostralis & - & - & - & 730 & field & $\begin{array}{l}\text { Kaneko (1989), sub } \\
\text { Archoplophora villosa, Japan }\end{array}$ \\
\hline \multicolumn{7}{|l|}{ Euphthiracaridae } \\
\hline Acrotritia clavata & - & - & $61-72$ & - & lab $/ 28 \pm 2^{\circ} \mathrm{C}$ & Syamjith \& Ramani (2020) \\
\hline Euphthiracarus sp. & - & - & 64 & - & $\mathrm{lab} / 25^{\circ} \mathrm{C}$ & Rohde (1955) \\
\hline Pseudotritia sp. & - & - & 64 & - & & Rohde (1955) \\
\hline \multicolumn{7}{|l|}{ Phthiracaridae } \\
\hline Steganacarus cf. striculus & & - & 730 & - & field $/ 0-15^{\circ} \mathrm{C}$ & Hagvar (1998) \\
\hline \multirow[t]{2}{*}{ Steganacarus magnus } & - & $\begin{array}{l}730- \\
1095\end{array}$ & 400 & - & $\mathrm{lab} / 18^{\circ} \mathrm{C}$ & Webb (1977) \\
\hline & $550-688$ & 950 & 400 & 500 & $\mathrm{lab} / 11.6-18^{\circ} \mathrm{C}$ & Webb (1989) \\
\hline \multicolumn{7}{|l|}{ Trhypochthoniidae } \\
\hline Allonothrus giganticus & - & - & $30-51$ & - & $\mathrm{lab} / 29 \pm 1^{\circ} \mathrm{C}$ & $\begin{array}{l}\text { Ramani \& Haq (1993), } \\
\text { different food }\end{array}$ \\
\hline \multirow[t]{4}{*}{ Archegozetes longisetosus } & - & - & $30-65$ & $60-228$ & $\mathrm{lab} / 23^{\circ} \mathrm{C}$ & Bergmann (2006) \\
\hline & - & $126-280$ & $32-88$ & - & $\mathrm{lab} / 28^{\circ} \mathrm{C}$ & $\begin{array}{l}\text { Seniczak (1998), } \\
\text { Brückner et al. (2018), } \\
\text { reared on different diets }\end{array}$ \\
\hline & - & - & $32-65$ & - & $\mathrm{lab} / 22-31^{\circ} \mathrm{C}$ & $\begin{array}{l}\text { Haq \& Adolph (1981), } \\
\text { Heethoff et al. (2007), } \\
\text { Honciuc (1996) }\end{array}$ \\
\hline & $10-88$ & - & $27-58$ & - & $\mathrm{lab} / 30^{\circ} \mathrm{C}$ & $\begin{array}{l}\text { Seniczak et al. }(1998,2005 \text {, } \\
\text { 2006, 2009), Seniczak \& } \\
\text { Seniczak (2002), tested } \\
\text { effects of toxic metals }\end{array}$ \\
\hline Mainothrus badius & - & - & $59-114$ & - & $\mathrm{lab} / 19-22.5^{\circ} \mathrm{C}$ & Ermilov (2008) \\
\hline $\begin{array}{l}\text { Trhypochthoniellus } \\
\text { brevisetus }\end{array}$ & - & $>1825$ & 1825 & - & field & $\begin{array}{l}\text { Kuriki (1995), Northeast } \\
\text { Japan mountainous area }\end{array}$ \\
\hline $\begin{array}{l}\text { Trhypochthoniellus } \\
\text { longisetus }\end{array}$ & - & - & $66-148$ & - & $\mathrm{lab} / 19-22.5^{\circ} \mathrm{C}$ & Ermilov (2008) \\
\hline $\begin{array}{l}\text { Trhypochthoniellus } \\
\text { crassus }\end{array}$ & - & $58-208$ & - & - & $\mathrm{lab} / 15-25^{\circ} \mathrm{C}$ & $\begin{array}{l}\text { Kuriki (2010), sub } \\
\text { Trhypochthoniellus crassus }\end{array}$ \\
\hline \multirow[t]{3}{*}{ Trhypochthonius tectorum } & - & - & $49-116$ & - & $\mathrm{lab} / 17-20^{\circ} \mathrm{C}$ & Ermilov et al. (2004) \\
\hline & - & - & $49-116$ & - & $\mathrm{lab} / 17-20^{\circ} \mathrm{C}$ & Ermilov (2008) \\
\hline & - & - & $50-77$ & - & $\mathrm{lab} / 20.6-27^{\circ} \mathrm{C}$ & $\begin{array}{l}\text { Taberly (1987) ex Grishina } \\
\text { (1991) }\end{array}$ \\
\hline \multicolumn{7}{|l|}{ Nothridae } \\
\hline Nothrus anaunensis & - & - & $172-296$ & - & $\mathrm{lab} / 20-23^{\circ} \mathrm{C}$ & Ermilov (2008) \\
\hline Nothrus biciliatus & - & - & $73-278$ & - & $\mathrm{lab} / 28^{\circ} \mathrm{C}$ & $\begin{array}{l}\text { Saichue et al. (1972), } \\
\text { reared on different diets }\end{array}$ \\
\hline Nothrus borussicus & - & - & $154-246$ & - & $\mathrm{lab} / 20-23^{\circ} \mathrm{C}$ & Ermilov (2008) \\
\hline Nothrus lasebikani & $4-6$ & - & - & - & $\mathrm{lab} / 28 \pm 2^{\circ} \mathrm{C}$ & $\begin{array}{l}\text { Badejo \& Akinwole (2007), } \\
\text { reared on different diets }\end{array}$ \\
\hline \multirow[t]{2}{*}{ Nothrus palustris } & - & - & $<150$ & - & $\mathrm{lab} / 18-20^{\circ} \mathrm{C}$ & Grishina (1997) \\
\hline & - & - & $148-174$ & - & field & $\begin{array}{l}\text { Shaldybina (1984), } \\
\text { Russia, Volga }\end{array}$ \\
\hline
\end{tabular}

...Continued on the next page 
TABLE 1. (Continued)

\begin{tabular}{|c|c|c|c|c|c|c|}
\hline Taxon & Longevity & Lifespan & Egg-adult & Egg-egg & Rearing & References and notes \\
\hline & - & $140-351$ & - & - & $\mathrm{lab} / 20^{\circ} \mathrm{C}$ & Lebrun (1970a) \\
\hline & 250 & - & 262 & - & $\mathrm{lab} / 18-20^{\circ} \mathrm{C}$ & Lebrun (1970a) \\
\hline & - & - & $147-148$ & - & $\mathrm{lab} / 18-20^{\circ} \mathrm{C}$ & $\begin{array}{l}\text { Shaldybina (1981) } \\
\text { ex Grishina (1991) }\end{array}$ \\
\hline \multirow[t]{3}{*}{ Nothrus silvestris } & 145 & 221 & 75 & 85 & lab / room temp. & Heba (2009) \\
\hline & - & - & 153 & - & $\mathrm{lab} / 25^{\circ} \mathrm{C}$ & Sengbusch (1958b) \\
\hline & - & - & $143-227$ & - & lab / $20-23^{\circ} \mathrm{C}$ & Ermilov (2008) \\
\hline \multicolumn{7}{|l|}{ Crotoniidae } \\
\hline \multirow[t]{2}{*}{ Camisia biurus } & - & - & $101-119$ & - & $\mathrm{lab} / 19-20^{\circ} \mathrm{C}$ & Ermilov (2007) \\
\hline & - & - & $101-193$ & - & $\mathrm{lab} / 17-19.5^{\circ} \mathrm{C}$ & Ermilov (2008) \\
\hline \multirow[t]{2}{*}{ Camisia segnis } & $200-336$ & - & $131-246$ & - & lab / room temp. & Grandjean (1950) \\
\hline & - & - & $90-169$ & - & $\mathrm{lab} / 17-19.5^{\circ} \mathrm{C}$ & Ermilov (2008) \\
\hline \multirow[t]{2}{*}{ Camisia spinifer } & - & - & 126 & - & $\mathrm{lab} / 25^{\circ} \mathrm{C}$ & Sengbusch (1958b) \\
\hline & - & - & $133-231$ & - & $\mathrm{lab} / 20-23^{\circ} \mathrm{C}$ & Ermilov (2008) \\
\hline Heminothrus longisetosus & - & - & $122-176$ & - & $\mathrm{lab} / 22.5^{\circ} \mathrm{C}$ & Ermilov (2008) \\
\hline Heminothrus thori & - & - & $159-174$ & - & $\mathrm{lab} / 22.5^{\circ} \mathrm{C}$ & Ermilov (2008) \\
\hline \multirow[t]{9}{*}{ Platynothrus peltifer } & - & - & $240-300$ & $\begin{array}{l}330- \\
365\end{array}$ & $\begin{array}{l}\text { field } / 5^{\circ} \mathrm{C} \text { mean } \\
\text { annual }\end{array}$ & Block (1965) \\
\hline & - & - & $114-303$ & - & $\mathrm{lab} / 20-27^{\circ} \mathrm{C}$ & Taberly (1988) \\
\hline & - & - & $117-150$ & - & $\mathrm{lab} / 15-20^{\circ} \mathrm{C}$ & $\begin{array}{l}\text { Grandjean (1950), } \\
\text { Hartenstein (1962d) }\end{array}$ \\
\hline & $156-212$ & $310-355$ & $143-154$ & - & lab / room temp. & Grandjean (1950) \\
\hline & - & 332 & - & - & lab / room temp. & Haarløv (1960) \\
\hline & $1-7$ & & & & $1 \mathrm{ab} / 5-25^{\circ} \mathrm{C}$ & $\begin{array}{l}\text { Madge (1964), } \\
\text { reaction to temperature and } \\
\text { relative humidity }\end{array}$ \\
\hline & 406 & 576 & 170 & - & $\mathrm{lab} / 25^{\circ} \mathrm{C}$ & Jalil (1972) \\
\hline & - & - & $330-365$ & - & lab $/ 3-15^{\circ} \mathrm{C}$ & Weigmann (1975) \\
\hline & - & - & $154-216$ & - & $\mathrm{lab} / 20.5-22.5^{\circ} \mathrm{C}$ & Ermilov (2008) \\
\hline Platynothrus skottsbergii & - & - & 365 & - & $\begin{array}{l}\text { field /subantarctic } \\
\text { climate }\end{array}$ & West (1982) \\
\hline \multicolumn{7}{|l|}{ Nanhermanniidae } \\
\hline $\begin{array}{l}\text { Nanhermannia } \mathrm{cf} . \\
\text { coronata }\end{array}$ & - & - & $112-149$ & - & $\mathrm{lab} / 20-22.5^{\circ} \mathrm{C}$ & Ermilov \& Łochyńska (2008) \\
\hline \multirow[t]{2}{*}{ Nanhermannia nana } & - & - & 83-144 & $88-170$ & $\mathrm{lab} / 25^{\circ} \mathrm{C}$ & Sengbusch (1958a) \\
\hline & $<2$ & & & & $\mathrm{lab} / 5-25^{\circ} \mathrm{C}$ & $\begin{array}{l}\text { Madge (1964), } \\
\text { reaction to temperature and } \\
\text { relative humidity }\end{array}$ \\
\hline \multicolumn{7}{|l|}{ Hermanniidae } \\
\hline Hermannia gibba & - & - & $205-280$ & $1.5-730$ & $\mathrm{lab} / 15-20^{\circ} \mathrm{C}$ & Bäumler (1970) \\
\hline Hermannia scabra & - & - & 185 & - & $\mathrm{lab} / 20^{\circ} \mathrm{C}$ & Jalil (1965) \\
\hline Hermannia subglabra & - & $540-600$ & $120-365$ & - & $\mathrm{lab} / 3-15^{\circ} \mathrm{C}$ & Weigmann (1975) \\
\hline \multicolumn{7}{|l|}{ Neoliodidae } \\
\hline Teleioliodes sp. & 102 & - & - & - & $\mathrm{lab} / 28 \pm 2^{\circ} \mathrm{C}$ & $\begin{array}{l}\text { Badejo \& Akinwole (2007), } \\
\text { reared on different diets }\end{array}$ \\
\hline Damaeidae & & & & & & \\
\hline
\end{tabular}


TABLE 1. (Continued)

\begin{tabular}{|c|c|c|c|c|c|c|}
\hline Taxon & Longevity & Lifespan & Egg-adult & Egg-egg & Rearing & References and notes \\
\hline \multirow[t]{3}{*}{$\begin{array}{l}\text { Damaeus (Adamaeus) } \\
\text { onustus }\end{array}$} & - & - & $>132$ & - & $\mathrm{lab} / 20.5^{\circ} \mathrm{C}$ & $\begin{array}{l}\text { Lebrun (1974), development } \\
\text { only to tritonymph }\end{array}$ \\
\hline & 634 & - & 140 & - & $\mathrm{lab} / 20-25^{\circ} \mathrm{C}$ & Pauly (1956) \\
\hline & $1-40$ & & & & $\mathrm{lab} / 5-25^{\circ} \mathrm{C}$ & $\begin{array}{l}\text { Madge (1964), sub Belba } \\
\text { geniculosa, reaction to } \\
\text { temperature and relative } \\
\text { humidity }\end{array}$ \\
\hline Belba corynopus & - & - & $63-327$ & - & $\mathrm{lab} / 15-20^{\circ} \mathrm{C}$ & Luxton (1981a) \\
\hline Belba meridionalis & - & $>365$ & 54 & - & $\mathrm{lab} / 25^{\circ} \mathrm{C}$ & Shereef (1972) \\
\hline Damaeus angustipes & 56 & - & 87 & - & $\mathrm{lab} / 20^{\circ} \mathrm{C}$ & $\begin{array}{l}\text { Hartenstein (1962a), } \\
\text { sub Belba kingi }\end{array}$ \\
\hline Damaeus auritus & - & - & $64-85$ & - & $\mathrm{lab} / 20-25^{\circ} \mathrm{C}$ & $\begin{array}{l}\text { Pauly (1956), } \\
\text { Sengbusch (1958b) }\end{array}$ \\
\hline \multirow[t]{3}{*}{ Damaeus clavipes } & - & - & $240-300$ & $\begin{array}{c}330- \\
365\end{array}$ & $\begin{array}{l}\text { field } / 5^{\circ} \mathrm{C} \text { mean } \\
\text { annual }\end{array}$ & $\begin{array}{l}\text { Block (1965), } \\
\text { field observations in } \\
\text { Westmorland England }\end{array}$ \\
\hline & - & - & - & $\begin{array}{c}240- \\
365\end{array}$ & field & $\begin{array}{l}\text { Luxton (1981a), } \\
\text { field observations in Denmark }\end{array}$ \\
\hline & - & - & $77-182$ & - & $\mathrm{lab} / 15-20.5^{\circ} \mathrm{C}$ & $\begin{array}{l}\text { Lebrun (1974), } \\
\text { Luxton (1981a) }\end{array}$ \\
\hline \multirow[t]{2}{*}{ Damaeus gracilipes } & - & - & 76 & - & $\mathrm{lab} / 20-25^{\circ} \mathrm{C}$ & Pauly (1956) \\
\hline & - & - & 130 & - & $\mathrm{lab} / 25^{\circ} \mathrm{C}$ & Sengbusch (1958b) \\
\hline Epidamaeus diversipilis & - & - & 120 & - & $\mathrm{lab} / 20^{\circ} \mathrm{C}$ & Schenker (1987) \\
\hline Epidamaeus grandjeani & - & - & $55-136$ & - & $\mathrm{lab} / 14-26^{\circ} \mathrm{C}$ & $\begin{array}{l}\text { Lyashchev (1984) } \\
\text { ex Grishina (1991) }\end{array}$ \\
\hline \multirow[t]{2}{*}{ Epidamaeus kamaensii } & - & & 105 & - & $\mathrm{lab} / 18-20^{\circ} \mathrm{C}$ & $\begin{array}{l}\text { Chistyakov \& Orlova (1979) } \\
\text { ex Grishina (1991) }\end{array}$ \\
\hline & & 230 & & & & Shereef (1972) \\
\hline Epidamaeus verrucatus & - & - & $25-44$ & - & $\mathrm{lab} / 17-25^{\circ} \mathrm{C}$ & Enami (1992) \\
\hline $\begin{array}{l}\text { Epidamaeus } \\
(\text { Akrodamaeus }) \mathrm{sp} .\end{array}$ & $180-240$ & $240-300$ & $63-67$ & - & $\mathrm{lab} / 20-25^{\circ} \mathrm{C}$ & $\begin{array}{l}\text { Estrada-Venegas \& Norton } \\
\text { (2001) }\end{array}$ \\
\hline Metabelba montana & - & - & 65 & - & $\mathrm{lab} / 20^{\circ} \mathrm{C}$ & Hartenstein (1962b) \\
\hline Spatiodamaeus boreus & - & - & 120 & - & $\mathrm{lab} / 16-20^{\circ} \mathrm{C}$ & $\begin{array}{l}\text { Sitnikova (1959), sub } \\
\text { Damaeus (Spatiodamaeus) } \\
\text { boreus ex Grishina (1991) }\end{array}$ \\
\hline $\begin{array}{l}\text { Spatiodamaeus } \\
\text { subverticillipes }\end{array}$ & - & 300 & $62-75$ & - & $\mathrm{lab} / 25^{\circ} \mathrm{C}$ & Shereef (1972) \\
\hline \multicolumn{7}{|l|}{ Cepheidae } \\
\hline Conoppia palmicincta & - & - & 375 & - & $\mathrm{lab} / 25^{\circ} \mathrm{C}$ & $\begin{array}{l}\text { Michael (1884), } \\
\text { Woodring \& Cook (1962) }\end{array}$ \\
\hline \multicolumn{7}{|l|}{ Peloppiidae } \\
\hline \multirow[t]{3}{*}{ Ceratoppia bipilis } & - & - & $43-65$ & - & $\mathrm{lab} / 17-20^{\circ} \mathrm{C}$ & Ermilov \& Łochyńska (2008) \\
\hline & - & - & 79 & - & $\mathrm{lab} / 25^{\circ} \mathrm{C}$ & $\begin{array}{l}\text { Michael (1884), } \\
\text { Woodring \& Cook } 1962\end{array}$ \\
\hline & - & - & $49-87$ & - & $\mathrm{lab} / 18^{\circ} \mathrm{C}$ & Taberly (1957) \\
\hline Ceratoppia quadridentata & - & - & $57-89$ & - & $\mathrm{lab} / 17-20^{\circ} \mathrm{C}$ & Ermilov \& Łochyńska (2008) \\
\hline \multicolumn{7}{|l|}{ Liacaridae } \\
\hline Adoristes ovatus & - & - & $1-730$ & - & $\begin{array}{c}\text { field } / 6^{\circ} \mathrm{C} \text { mean } \\
\text { annual }\end{array}$ & Gourbière et al. (1985) \\
\hline
\end{tabular}

...Continued on the next page 
TABLE 1. (Continued)

\begin{tabular}{|c|c|c|c|c|c|c|}
\hline Taxon & Longevity & Lifespan & Egg-adult & Egg-egg & Rearing & References and notes \\
\hline & - & - & 730 & - & field $/ 0-15^{\circ} \mathrm{C}$ & Hagvar (1998) \\
\hline & - & - & $105-137$ & - & $\mathrm{lab} / 18-22^{\circ} \mathrm{C}$ & Travnicek (1989) \\
\hline Liacarus coracinus & - & - & $97-141$ & - & $\mathrm{lab} / 18-22^{\circ} \mathrm{C}$ & Travnicek (1989) \\
\hline Liacarus nitens & - & - & $119-140$ & - & $\mathrm{lab} / 18-22^{\circ} \mathrm{C}$ & Travnicek (1989) \\
\hline Liacarus subterraneus & - & - & $113-146$ & - & $\mathrm{lab} / 18-22^{\circ} \mathrm{C}$ & Travnicek (1989) \\
\hline Dorycranosus acutus & - & - & $121-135$ & - & $\mathrm{lab} / 18-22^{\circ} \mathrm{C}$ & $\begin{array}{l}\text { Travnicek (1989), sub } \\
\text { Dorycranosus infissus }\end{array}$ \\
\hline Dorycranosus moraviacus & - & - & $104-139$ & - & $\mathrm{lab} / 18-22^{\circ} \mathrm{C}$ & Travnicek (1989) \\
\hline Xenillus clypeator & - & - & $117-143$ & - & $\mathrm{lab} / 18-22^{\circ} \mathrm{C}$ & Travnicek (1989) \\
\hline Xenillus tegeocranus & - & - & $116-146$ & - & $\mathrm{lab} / 18-22^{\circ} \mathrm{C}$ & Travnicek (1989) \\
\hline \multicolumn{7}{|l|}{ Eremobelbidae } \\
\hline Eremobelba geographica & - & 270 & 65 & - & $\mathrm{lab} / 25^{\circ} \mathrm{C}$ & Shereef (1972) \\
\hline Eremobelba nervosa & - & - & $68-75$ & - & $\mathrm{lab} / 20^{\circ} \mathrm{C}$ & Hartenstein (1962b) \\
\hline \multicolumn{7}{|l|}{ Thyrisomidae } \\
\hline Oribella paolii & - & - & - & 365 & field & $\begin{array}{l}\text { Luxton (1981a), } \\
\text { field observations in Denmark }\end{array}$ \\
\hline \multicolumn{7}{|l|}{ Oppiidae } \\
\hline Lanceoppia nodosa & - & - & $14-54$ & $14-54$ & $\mathrm{lab} / 16-32^{\circ} \mathrm{C}$ & $\begin{array}{l}\text { Bhattacharya et al. (1978), } \\
\text { sub Oppia nodosa }\end{array}$ \\
\hline Multioppia bayoumii & - & - & $18-31$ & - & $\mathrm{lab} / 25^{\circ} \mathrm{C}$ & $\begin{array}{l}\text { Shereef \& Zaher (1980), } \\
\text { reared on different diets }\end{array}$ \\
\hline \multirow[t]{2}{*}{ Multioppia wilsoni } & $>365$ & - & $18-21$ & 25 & $\mathrm{lab} / 25 \pm 1^{\circ} \mathrm{C}$ & Shereef (1976a) \\
\hline & & - & $16-23$ & - & $\mathrm{lab} / 25^{\circ} \mathrm{C}$ & Shereef (1976a) \\
\hline Niloppia sticta & $>365$ & - & $11-16$ & 19 & $\mathrm{lab} / 25 \pm 1^{\circ} \mathrm{C}$ & $\begin{array}{l}\text { Shereef }(1976 a) \\
\text { sub Oppia sticta }\end{array}$ \\
\hline Oppia denticulata & - & $300-425$ & $21-33$ & - & $\mathrm{lab} / 18-25^{\circ} \mathrm{C}$ & $\begin{array}{l}\text { Nannelli (1975), sub Oppia } \\
\text { concolor ex Luxton (1981a), } \\
\text { Seniczak et al. (2017) }\end{array}$ \\
\hline \multirow[t]{2}{*}{ Oppia nitens } & - & - & 40 & - & lab / room temp. & Michael (1884) \\
\hline & - & - & $45-46$ & 105 & $\mathrm{lab} / 20^{\circ} \mathrm{C}$ & $\begin{array}{l}\text { Sengbusch \& Sengbusch } \\
\text { (1970) }\end{array}$ \\
\hline \multirow[t]{3}{*}{ Oppiella nova } & - & - & $23-60$ & ca. 180 & $\mathrm{lab} / 15-25^{\circ} \mathrm{C}$ & Kaneko (1988a) \\
\hline & 30 & 60 & 23 & 30 & $\mathrm{lab} / 25^{\circ} \mathrm{C}$ & $\begin{array}{l}\text { Woodring \& Cook (1962), } \\
\text { sub Oppia neerlandica }\end{array}$ \\
\hline & - & - & $24-36$ & - & $\mathrm{lab} / 18.5-25^{\circ} \mathrm{C}$ & $\begin{array}{l}\text { Chistyakov (1970) } \\
\text { ex Grishina (1991) }\end{array}$ \\
\hline \multicolumn{7}{|l|}{ Granuloppiidae } \\
\hline Granuloppia sp. & - & 240 & 34 & - & $\mathrm{lab} / 25^{\circ} \mathrm{C}$ & Shereef (1972) \\
\hline \multicolumn{7}{|l|}{ Carabodidae } \\
\hline \multirow[t]{2}{*}{ Carabodes labyrinthicus } & - & - & 730 & - & $\begin{array}{c}\text { field } \\
\text { (arboricolous) }\end{array}$ & Wunderle 1991 \\
\hline & $>365$ & - & - & - & lab / room temp. & Block (1965) \\
\hline Carabodes polyporetes & - & - & $70-84$ & - & lab / room temp. & Reeves (1991) \\
\hline \multicolumn{7}{|l|}{ Tectocepheidae } \\
\hline Tectocepheus sarekensis & - & - & 102 & - & $\mathrm{lab} / 25^{\circ} \mathrm{C}$ & Murphy \& Jalil (1964) \\
\hline \multirow[t]{2}{*}{ Tectocepheus velatus } & - & - & 102 & - & $\mathrm{lab} / 25^{\circ} \mathrm{C}$ & Murphy \& Jalil (1964) \\
\hline & - & - & $57-72$ & - & $\mathrm{lab} / 16-20^{\circ} \mathrm{C}$ & Grishina (1991) \\
\hline
\end{tabular}

...Continued on the next page 
TABLE 1. (Continued)

\begin{tabular}{|c|c|c|c|c|c|c|}
\hline Taxon & Longevity & Lifespan & Egg-adult & Egg-egg & Rearing & References and notes \\
\hline & - & - & $>730$ & - & field & Solhøy (1975), Norway \\
\hline \multicolumn{7}{|l|}{ Hydrozetidae } \\
\hline Hydrozetes lemnae & - & - & $56-57$ & - & $\mathrm{lab} / 20.6^{\circ} \mathrm{C}$ & Ermilov (2006) \\
\hline \multicolumn{7}{|l|}{ Ameronothridae } \\
\hline Ameronothrus lapponicus & - & - & $\begin{array}{l}1460- \\
2190\end{array}$ & - & field & $\begin{array}{l}\text { Tilrem (1994), South Norway, } \\
\text { Hardangervidda }\end{array}$ \\
\hline \multirow[t]{3}{*}{ Ameronothrus lineatus } & $730-1095$ & $>1825$ & $\begin{array}{l}1095- \\
2920\end{array}$ & ca. 1825 & lab / polar climate & $\begin{array}{l}\text { Søvik \& Leinaas (2003a, b), } \\
\text { Svalbard }\end{array}$ \\
\hline & $730-1095$ & $\begin{array}{c}2190- \\
2920\end{array}$ & $\begin{array}{l}1095- \\
2920\end{array}$ & ca. 1825 & $\begin{array}{l}\text { field / polar } \\
\text { climate }\end{array}$ & Søvik et al. (2003), Svalbard \\
\hline & - & $<365$ & $150-270$ & - & field & $\begin{array}{l}\text { Bücking et al. (1998), } \\
\text { German North Sea coast }\end{array}$ \\
\hline Ameronothrus maculatus & - & ca. 730 & ca. 365 & - & field & $\begin{array}{l}\text { Bücking et al. (1998), } \\
\text { German North Sea coast }\end{array}$ \\
\hline Ameronothrus marinus & - & ca. 730 & ca. 365 & - & field & $\begin{array}{l}\text { Bücking et al. (1998), } \\
\text { German North Sea coast }\end{array}$ \\
\hline Ameronothrus schneideri & - & - & 330 & - & $\mathrm{lab} / 3-15^{\circ} \mathrm{C}$ & $\begin{array}{l}\text { Weigmann (1975), } \\
\text { German North Sea coast }\end{array}$ \\
\hline \multicolumn{7}{|l|}{ Podacaridae } \\
\hline Alaskozetes antarcticus & $>730$ & $\begin{array}{c}2190- \\
2555\end{array}$ & - & $>1825$ & $\begin{array}{l}\text { field / polar } \\
\text { climate }\end{array}$ & $\begin{array}{l}\text { Convey (1994), } \\
\text { Block \& Convey (1995) }\end{array}$ \\
\hline \multicolumn{7}{|l|}{ Cymbaeremaeidae } \\
\hline Cymbaeremaeus cymba & - & - & 365 & - & $\begin{array}{c}\text { field } \\
\text { (arboricolous) }\end{array}$ & Wunderle (1991) \\
\hline \multicolumn{7}{|l|}{ Micreremidae } \\
\hline Micreremus brevipes & - & - & 365 & - & $\begin{array}{c}\text { field } \\
\text { (arboricolous) }\end{array}$ & Wunderle (1991) \\
\hline \multicolumn{7}{|l|}{ Scutoverticidae } \\
\hline Scutovertex perforatus & - & - & $66-85$ & - & $\mathrm{lab} / 23^{\circ} \mathrm{C}$ & Ermilov et al. (2008) \\
\hline Scutovertex sculptus & - & - & $72-135$ & - & $\mathrm{lab} / 18-23^{\circ} \mathrm{C}$ & $\begin{array}{l}\text { Ermilov et al. (2008), } \\
\text { sub Scutovertex rugosus }\end{array}$ \\
\hline \multicolumn{7}{|l|}{ Phenopelopidae } \\
\hline Eupelops torulosus & - & - & 180 & - & $\mathrm{lab} / 18-21^{\circ} \mathrm{C}$ & $\begin{array}{l}\text { Sitnikova (1969) ex Grishina } \\
\text { (1991) }\end{array}$ \\
\hline \multicolumn{7}{|l|}{ Achipteriidae } \\
\hline Achipteria coleptrata & - & - & - & 730 & field & $\begin{array}{l}\text { Luxton (1981a), } \\
\text { field observations in Denmark }\end{array}$ \\
\hline Achipteria holomonensis & - & - & 261 & - & $\mathrm{lab} / 25^{\circ} \mathrm{C}$ & Stamou (1989) \\
\hline Parachipteria punctata & - & - & $96-111$ & - & $\mathrm{lab} / 22^{\circ} \mathrm{C}$ & $\begin{array}{l}\text { Chistyakov (1984) } \\
\text { ex Grishina (1991) }\end{array}$ \\
\hline $\begin{array}{l}\text { Plakoribates } \\
\text { multicuspidus }\end{array}$ & 313 & - & $81-93$ & 103 & $\mathrm{lab} / 25^{\circ} \mathrm{C}$ & Shereef (1977) \\
\hline \multicolumn{7}{|l|}{ Oribatellidae } \\
\hline Oribatella berlesei & - & - & 107 & - & $\mathrm{lab} / 16^{\circ} \mathrm{C}$ & $\begin{array}{l}\text { Chistyakov (1981) } \\
\text { ex Grishina (1991) }\end{array}$ \\
\hline \multicolumn{7}{|l|}{ Limnozetidae } \\
\hline Limnozetes ciliatus & - & $78-142$ & - & - & $\mathrm{lab} / 15-25^{\circ} \mathrm{C}$ & Kuriki (2008) \\
\hline Ceratozetidae & & & & & & \\
\hline
\end{tabular}

...Continued on the next page 
TABLE 1. (Continued)

\begin{tabular}{|c|c|c|c|c|c|c|}
\hline Taxon & Longevity & Lifespan & Egg-adult & Egg-egg & Rearing & References and notes \\
\hline Ceratozetes cisalpinus & $>330-390$ & - & 32 & $47-52$ & $\mathrm{lab} / 25^{\circ} \mathrm{C}$ & Woodring \& Cook (1962) \\
\hline \multirow[t]{5}{*}{ Ceratozetes gracilis } & - & - & 41 & - & lab / room temp. & Haarløv (1960) \\
\hline & - & - & $119-149$ & - & $\mathrm{lab} / 20^{\circ} \mathrm{C}$ & Hartenstein (1962c) \\
\hline & - & - & - & $>730$ & field & $\begin{array}{l}\text { Luxton (1981a), } \\
\text { field observations in Denmark }\end{array}$ \\
\hline & - & ca. 4.730 & 540 & - & $\mathrm{lab} / 15^{\circ} \mathrm{C}$ & Mitchell (1977b) \\
\hline & & - & $54-113$ & - & lab / room temp. & $\begin{array}{l}\text { Shaldybina (1964) } \\
\text { ex Grishina (1991) }\end{array}$ \\
\hline Ceratozetes kananaskis & ca. 4.730 & - & 465 & - & $\mathrm{lab} / 15^{\circ} \mathrm{C}$ & Mitchell (1977b) \\
\hline Ceratozetes mediocris & - & - & $51-69$ & - & lab / room temp. & $\begin{array}{l}\text { Shaldybina (1967) } \\
\text { ex Grishina (1991) }\end{array}$ \\
\hline Ceratozetes minimus & - & - & $39-60$ & - & lab / room temp. & $\begin{array}{l}\text { Shaldybina (1964) } \\
\text { ex Grishina (1991) }\end{array}$ \\
\hline Ceratozetes virginicus & - & - & 53 & - & $\mathrm{lab} / 25^{\circ} \mathrm{C}$ & $\begin{array}{l}\text { Rockett \& Woodring (1966), } \\
\text { sub Ceratozetes jeweli }\end{array}$ \\
\hline Edwardzetes elongatus & - & - & $>730$ & - & $\begin{array}{c}\text { field / subantarctic } \\
\text { climate }\end{array}$ & West (1982) \\
\hline Euzetes globulus & - & - & $59-77$ & - & $\mathrm{lab} / 18-20^{\circ} \mathrm{C}$ & $\begin{array}{l}\text { Shaldybina (1973) } \\
\text { ex Grishina (1991) }\end{array}$ \\
\hline \multirow[t]{2}{*}{ Fuscozetes fuscipes } & - & - & $60-112$ & - & lab / room temp. & $\begin{array}{l}\text { Shaldybina (1978) } \\
\text { ex Grishina (1991) }\end{array}$ \\
\hline & $1-26$ & & & & $\mathrm{lab} / 5-25^{\circ} \mathrm{C}$ & $\begin{array}{l}\text { Madge (1964), } \\
\text { reaction to temperature and } \\
\text { relative humidity }\end{array}$ \\
\hline Oromurcia sudetica & $432-783$ & $\begin{array}{c}1095- \\
2190\end{array}$ & $730-1460$ & $\begin{array}{l}1095- \\
2190\end{array}$ & $\mathrm{lab} / 8-20^{\circ} \mathrm{C}$ & $\begin{array}{l}\text { Schatz }(1985) \text { reared under } \\
\text { high alpine conditions }\end{array}$ \\
\hline Sphaerozetes orbicularis & - & - & $127-133$ & - & lab / room temp. & $\begin{array}{l}\text { Shaldybina (1968) } \\
\text { ex Grishina (1991) }\end{array}$ \\
\hline Trichoribates berlesei & - & - & $49-75$ & - & $\mathrm{lab} / 20-22^{\circ} \mathrm{C}$ & $\begin{array}{l}\text { Shaldybina (1960), } \\
\text { sub T. trimaculatus ex } \\
\text { Grishina (1991) }\end{array}$ \\
\hline Zetomimus furcatus & - & - & $30-61$ & - & $\mathrm{lab} / 18-20^{\circ} \mathrm{C}$ & $\begin{array}{l}\text { Shaldybina (1969) } \\
\text { ex Grishina (1991) }\end{array}$ \\
\hline \multicolumn{7}{|l|}{ Chamobatidae } \\
\hline Chamobates cuspidatus & - & - & - & 365 & field & $\begin{array}{l}\text { Luxton (1981a), } \\
\text { field observations in Denmark }\end{array}$ \\
\hline Chamobates spinosus & - & - & $44-48$ & - & lab & $\begin{array}{l}\text { Shaldybina (1966) } \\
\text { ex Grishina (1991) }\end{array}$ \\
\hline Chamobates subglobulus & - & - & $71-124$ & - & $\mathrm{lab} / 18-20^{\circ} \mathrm{C}$ & $\begin{array}{l}\text { Shaldybina (1971) } \\
\text { ex Grishina (1991) }\end{array}$ \\
\hline \multicolumn{7}{|l|}{ Maudheimiidae } \\
\hline Maudheimia wilsoni & - & $4-1825$ & - & 1095 & $\begin{array}{l}\text { field / antarctic } \\
\text { climate }\end{array}$ & Marshall \& Convey (1999) \\
\hline \multicolumn{7}{|l|}{ Humerobatidae } \\
\hline \multirow{2}{*}{$\begin{array}{l}\text { Humerobates } \\
\text { rostrolamellatus }\end{array}$} & 124 & 221 & 97 & - & $\mathrm{lab} / 25^{\circ} \mathrm{C}$ & Jalil (1969) \\
\hline & $4-57$ & & & & $\mathrm{lab} / 5-25^{\circ} \mathrm{C}$ & $\begin{array}{l}\text { Madge (1964), } \\
\text { reaction to temperature and } \\
\text { relative humidity }\end{array}$ \\
\hline Punctoribatidae & & & & & & \\
\hline
\end{tabular}

...Continued on the next page 
TABLE 1. (Continued)

\begin{tabular}{|c|c|c|c|c|c|c|}
\hline Taxon & Longevity & Lifespan & Egg-adult & Egg-egg & Rearing & References and notes \\
\hline \multirow[t]{2}{*}{ Minunthozetes semirufus } & - & - & 38 & - & $\mathrm{lab} / 25^{\circ} \mathrm{C}$ & Sengbusch (1958b) \\
\hline & - & - & $57-76$ & - & lab & $\begin{array}{l}\text { Shaldybina (1965) } \\
\text { ex Grishina (1991) }\end{array}$ \\
\hline $\begin{array}{l}\text { Paralamellobates } \\
\text { bengalensis }\end{array}$ & - & - & 27 & - & $\mathrm{lab} / 29 \pm 1^{\circ} \mathrm{C}$ & Haq \& Ramani (1984) \\
\hline Punctoribates hexagonus & - & - & $31-38$ & - & lab & $\begin{array}{l}\text { Shaldybina (1967) } \\
\text { ex Grishina (1991) }\end{array}$ \\
\hline Punctoribates punctum & - & - & $70-81$ & - & lab & $\begin{array}{l}\text { Shaldybina (1965) } \\
\text { ex Grishina (1991) }\end{array}$ \\
\hline \multicolumn{7}{|l|}{ Mochlozetidae } \\
\hline \multirow[t]{2}{*}{$\begin{array}{l}\text { Drymobatoides } \\
\text { malabaricus }\end{array}$} & - & - & $17-19$ & ca. 60 & $\mathrm{lab} / 30 \pm 1^{\circ} \mathrm{C}$ & $\begin{array}{l}\text { Adolph \& Haq (1984), } \\
\text { sub Pelokylla malabarica }\end{array}$ \\
\hline & - & - & 26 & - & field $/ 25 \pm 1^{\circ} \mathrm{C}$ & $\begin{array}{l}\text { Adolph \& Haq (1984), } \\
\text { sub Pelokylla malabarica }\end{array}$ \\
\hline Uracrobates indicus & - & - & 33 & 50 & $\mathrm{lab} / 31 \pm 1^{\circ} \mathrm{C}$ & Ramani \& Haq (1988) \\
\hline \multicolumn{7}{|l|}{ Oribatulidae } \\
\hline Jornadia larreae & - & 365 & $28-42$ & - & field & $\begin{array}{l}\text { Wallwork et al. (1986), } \\
\text { New Mexico, influence of } \\
\text { rainfall }\end{array}$ \\
\hline \multicolumn{7}{|l|}{ Scheloribatidae } \\
\hline Hemileius initialis & & - & 730 & - & field & $\begin{array}{l}\text { Luxton (1981a), } \\
\text { field observations in Denmark }\end{array}$ \\
\hline Muliercula inexpectata & $48-52$ & - & - & - & $\mathrm{lab} / 28 \pm 2^{\circ} \mathrm{C}$ & $\begin{array}{l}\text { Badejo \& Akinwole (2007), } \\
\text { reared on different diets }\end{array}$ \\
\hline Scheloribates decarinatus & - & - & $29-38$ & $40-53$ & $\mathrm{lab} / 26-32^{\circ} \mathrm{C}$ & Ramani \& Haq (1987) \\
\hline \multirow[t]{7}{*}{ Scheloribates laevigatus } & - & - & $42-115$ & - & $\mathrm{lab} / 25^{\circ} \mathrm{C}$ & Cleat (1952) \\
\hline & - & $109-158$ & $21-28$ & $32-38$ & $\mathrm{lab} / 25-30^{\circ} \mathrm{C}$ & Elmoghazy et al. (2012) \\
\hline & - & - & 28 & - & $\mathrm{lab} / 27.8-32.7^{\circ} \mathrm{C}$ & Narsapur (1983) \\
\hline & 120 & 180 & 64 & 84 & $\mathrm{lab} / 25^{\circ} \mathrm{C}$ & Woodring \& Cook (1962) \\
\hline & - & - & 179 & - & $\mathrm{lab} / 5^{\circ} \mathrm{C}$ & Woodring \& Cook (1962) \\
\hline & - & - & 70 & - & $\mathrm{lab} / 20-24^{\circ} \mathrm{C}$ & $\begin{array}{l}\text { Subbotina (1967) } \\
\text { ex Grishina (1991) }\end{array}$ \\
\hline & - & - & $66-185$ & - & $\mathrm{lab} / 15-25^{\circ} \mathrm{C}$ & $\begin{array}{l}\text { Subbotina }(1948,1970) \\
\text { ex Grishina }(1991)\end{array}$ \\
\hline Scheloribates latipes & - & - & $84-85$ & - & $\mathrm{lab} / 18-23^{\circ} \mathrm{C}$ & $\begin{array}{l}\text { Subbotina (1967), } \\
\text { Soldatova (1948) ex Grishina } \\
\text { (1991) }\end{array}$ \\
\hline $\begin{array}{l}\text { Scheloribates } \\
\text { mochlosimilaris }\end{array}$ & $26-46$ & - & - & - & $\mathrm{lab} / 28 \pm 2^{\circ} \mathrm{C}$ & $\begin{array}{l}\text { Badejo \& Akinwole (2007), } \\
\text { reared on different diets }\end{array}$ \\
\hline $\begin{array}{l}\text { Scheloribates (Hemileius) } \\
\text { nicki }\end{array}$ & - & - & $31-33$ & $31-33$ & lab / room temp. & $\begin{array}{l}\text { Denmark \& Woodring (1965), } \\
\text { sub Hemileius nicki }\end{array}$ \\
\hline Scheloribates nudus & - & - & $14-24$ & $25-35$ & $\mathrm{lab} / 23^{\circ} \mathrm{C}$ & Woodring (1965) \\
\hline Scheloribates parabilis & - & - & $14-24$ & $25-35$ & $\mathrm{lab} / 23^{\circ} \mathrm{C}$ & Woodring (1965) \\
\hline \multicolumn{7}{|l|}{ Haplozetidae } \\
\hline $\begin{array}{l}\text { Protoribates } \\
\text { lophothrichus }\end{array}$ & - & - & 150 & - & $\mathrm{lab} / 25^{\circ} \mathrm{C}$ & $\begin{array}{l}\text { Hartenstein (1962e), } \\
\text { sub Protoribates lophotrichus }\end{array}$ \\
\hline Protoribates seminudus & & & $18-23$ & - & $\mathrm{lab} / 28 \pm 1^{\circ} \mathrm{C}$ & $\begin{array}{l}\text { Ramani (1999), } \\
\text { sub Xylobates seminudus }\end{array}$ \\
\hline
\end{tabular}

... Continued on the next page 
TABLE 1. (Continued)

\begin{tabular}{|c|c|c|c|c|c|c|}
\hline Taxon & Longevity & Lifespan & Egg-adult & Egg-egg & Rearing & References and notes \\
\hline $\begin{array}{r}\text { Protoribates } \\
\text { souchnaiensis }\end{array}$ & $>365$ & - & $86-108$ & 123 & $\mathrm{lab} / 25^{\circ} \mathrm{C}$ & $\begin{array}{l}\text { Shereef (1977), } \\
\text { sub Xylobates souchnaiensis }\end{array}$ \\
\hline Rostrozetes ovulum & $90-210$ & $120-240$ & $35-45$ & - & $\mathrm{lab} / 23^{\circ} \mathrm{C}$ & $\begin{array}{l}\text { Woodring (1965), } \\
\text { sub Rostrozetes flavus ex } \\
\text { Luxton (1981a) }\end{array}$ \\
\hline \multicolumn{7}{|l|}{ Parakalummidae } \\
\hline Neoribates gracilis & 730 & $>730$ & $31-53$ & - & $\mathrm{lab} / 18-30^{\circ} \mathrm{C}$ & Travé \& Duran (1971)- \\
\hline \multicolumn{7}{|l|}{ Galumnidae } \\
\hline \multirow[t]{2}{*}{ Acrogalumna longipluma } & - & - & 25 & - & $\mathrm{lab} / 30 \pm 1^{\circ} \mathrm{C}$ & $\begin{array}{l}\text { Haq \& Adolph (1981), } \\
\text { sub Galumna longipluma }\end{array}$ \\
\hline & - & - & $61-66$ & - & $\mathrm{lab} / 25^{\circ} \mathrm{C}$ & $\begin{array}{l}\text { Sengbusch (1954, 1958b), } \\
\text { sub Galumna longipluma }\end{array}$ \\
\hline Galumna flabellifera & & & $48-67$ & - & $\mathrm{lab} / 25^{\circ} \mathrm{C}$ & Reddy et al. (1978) \\
\hline $\begin{array}{l}\text { Galumna flabellifera } \\
\text { orientalis }\end{array}$ & - & - & 25 & - & $\mathrm{lab} / 30 \pm 1^{\circ} \mathrm{C}$ & Haq \& Adolph (1981) \\
\hline Galumna ithacensis & - & - & 87 & - & $\mathrm{lab} / 25^{\circ} \mathrm{C}$ & $\begin{array}{l}\text { Sengbusch (1954), } \\
\text { sub Galumna elimata } \\
\text { ithacensis }\end{array}$ \\
\hline Galumna louisianae & $60-180$ & $120-240$ & $46-50$ & $74-78$ & $\mathrm{lab} / 23^{\circ} \mathrm{C}$ & $\begin{array}{l}\text { Woodring (1965), } \\
\text { sub Galumna confusa ex } \\
\text { Luxton (1981a) }\end{array}$ \\
\hline Galumna parva & $60-120$ & $90-150$ & $33-41$ & - & $\mathrm{lab} / 23^{\circ} \mathrm{C}$ & $\begin{array}{l}\text { Woodring (1965) } \\
\text { ex Luxton (1981a) }\end{array}$ \\
\hline Galumna unica & & - & 34 & - & $\mathrm{lab} / 28^{\circ} \mathrm{C}$ & $\begin{array}{l}\text { Haq \& Shereef (1992), } \\
\text { sub Galumna uniqua }\end{array}$ \\
\hline Galumna triquetra & - & - & $30-39$ & $42-59$ & $\mathrm{lab} / 27 \pm 1^{\circ} \mathrm{C}$ & Shereef \& Haq (1992) \\
\hline Galumna sp. & 365 & - & $65-94$ & - & $\mathrm{lab} / 25^{\circ} \mathrm{C}$ & $\begin{array}{l}\text { Stunkard (1944), } \\
\text { intermediate host of Moniezia } \\
\text { expansa }\end{array}$ \\
\hline Pergalumna ekaterinae & & - & 48 & - & $\mathrm{lab} / 26.4^{\circ} \mathrm{C}$ & Paez et al. (2019) \\
\hline Pergalumna emarginata & - & $>210$ & 42 & - & $\mathrm{lab} / 25^{\circ} \mathrm{C}$ & $\begin{array}{l}\text { Rockett \& Woodring (1966), } \\
\text { sub Pergalumna omniphagous }\end{array}$ \\
\hline \multirow[t]{4}{*}{ Pergalumna nervosa } & - & - & $43-63$ & - & $\mathrm{lab} / 20-25^{\circ} \mathrm{C}$ & $\begin{array}{l}\text { Sengbusch }(1954,1958 b) \\
\text { sub Galumna nervosus }\end{array}$ \\
\hline & $37-121$ & - & $33-51$ & - & $\mathrm{lab} / 30^{\circ} \mathrm{C}$ & $\begin{array}{l}\text { Seniczak (2007), } \\
\text { tested effect of copper }\end{array}$ \\
\hline & - & - & 47 & - & $\mathrm{lab} / 25^{\circ} \mathrm{C}$ & $\begin{array}{l}\text { Sengbusch (1954), } \\
\text { Woodring \& Cook (1962) }\end{array}$ \\
\hline & - & - & $110-158$ & - & $\mathrm{lab} / 16-18^{\circ} \mathrm{C}$ & $\begin{array}{l}\text { Chistyakov \& Orlova (1982) } \\
\text { ex Grishina (1991) }\end{array}$ \\
\hline Pergalumna sp. & - & - & $27-50$ & - & $\mathrm{lab} / 20-30^{\circ} \mathrm{C}$ & Tanimoto (1980) \\
\hline Pergalumna sp. & $46-287$ & - & - & - & lab / $28 \pm 2^{\circ} \mathrm{C}$ & $\begin{array}{l}\text { Badejo \& Akinwole (2007), } \\
\text { reared on different diets }\end{array}$ \\
\hline Orthogalumna terebrantis & - & $54-94$ & - & - & $\mathrm{lab} / 25^{\circ} \mathrm{C}$ & Cordo \& DeLoach (1975) \\
\hline \multicolumn{7}{|l|}{ Galumnellidae } \\
\hline Galumnella sp. & 12 & - & - & - & lab / $28 \pm 2^{\circ} \mathrm{C}$ & $\begin{array}{l}\text { Badejo \& Akinwole (2007), } \\
\text { sub Galumnella sonpona } \\
\text { (nomen nudum), reared on } \\
\text { different diets }\end{array}$ \\
\hline
\end{tabular}




\section{Results}

Table 1 lists the results of 210 investigations in 135 publications with data on life cycles (generation time and/or developmental time, 128 publications) and on lifespans (50 publications) of 153 oribatid species belonging to 45 families. Up to the present, the life cycles of 144 oribatid mite species from 42 families have been studied, most species from the families Damaeidae (15 spp.), Ceratozetidae, Galumnidae (13 spp. each), Liacaridae (8 spp.), Crotoniidae, Oppiidae, Scheloribatidae (7 spp. each), Trhypochthoniidae (6 spp.). The lifespan of 52 species ( 25 families) has been observed or estimated, mainly from the families Galumnidae ( $7 \mathrm{spp}$.), Damaeidae (5 spp.), Oppiidae (4 spp.), and Ceratozetidae, Nothridae, Scheloribatidae, Trhypochthoniidae (3 spp. each). The duration of the particular immature stages was not taken into account here.

The majority of life cycle studies on oribatid mites were carried out before 1990 (107 spp.) (Fig. 1). After this period the number of studies declined. During the last three decades 38 further species were studied, and 23 additional investigations concerned already studied species. Similarly, most lifespan observations were published before 1990 (33 spp.), with 19 additional species in the later years. Since 1990, further studies on 11 species with already known lifespans were carried out, mainly on Archegozetes longisetosus (see below). Most investigations (178) were done in the laboratory, some performed in the field and/or compared with the field data (31 investigations). Earlier studies considered the morphology and taxonomic assignment of immature stages to certain species and were performed at room temperature in the laboratory. From around the middle of the previous century, most investigations concentrated on the influence of temperature on the duration of development, the food quality and other parameters influencing life cycle, longevity or mortality.

\section{number of studies}

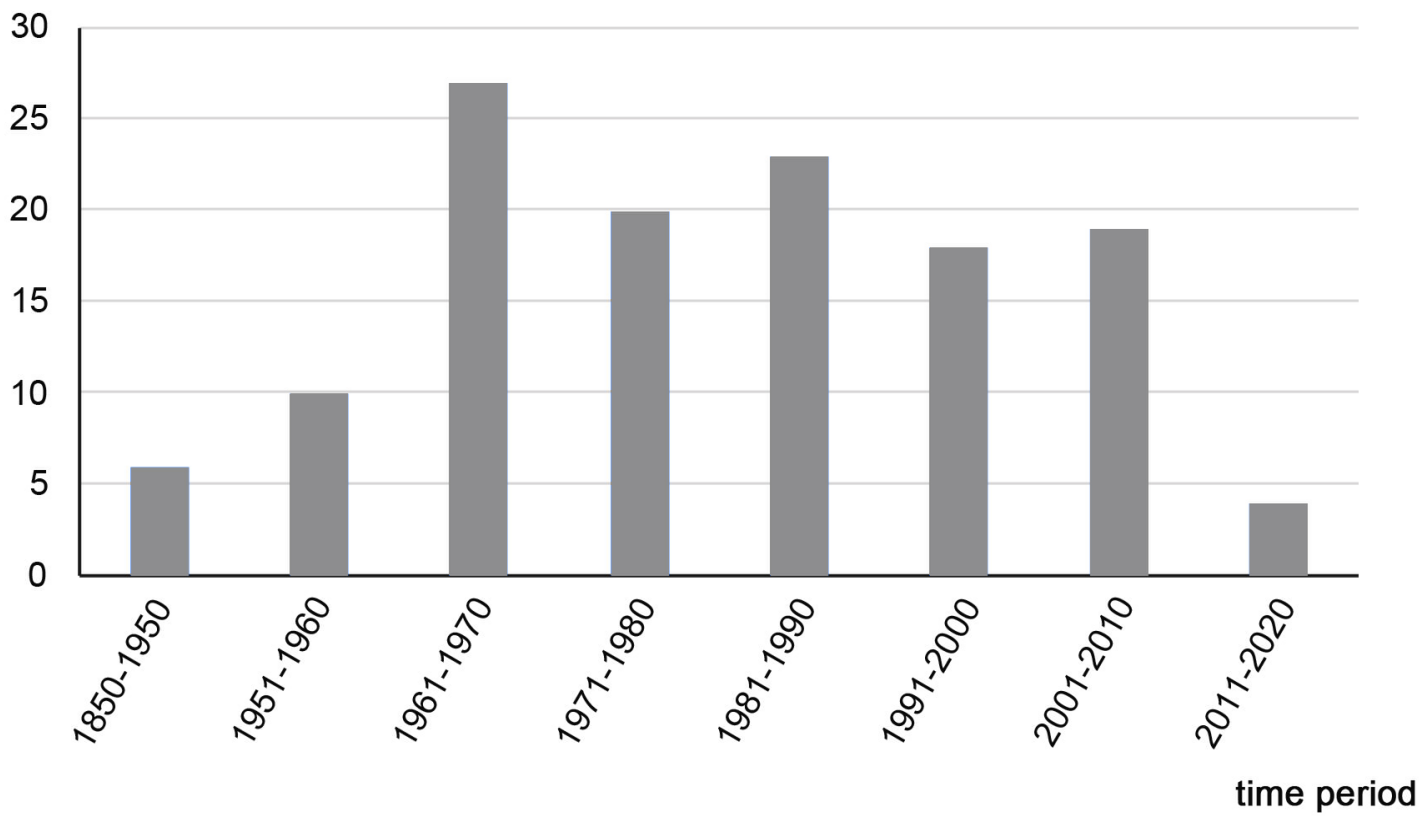

FIGURE 1. The number of studies on life history and lifespans in oribatid mites performed during 1850-2020, indicating a strong decline in the last decade.

Compared to the total known number of oribatid mite species, the studies on immatures and life cycles or the total lifespan are very sparse. Of all currently known oribatid species, juvenile instars of less than $8 \%$ of the species are described (Norton \& Ermilov 2014), the duration of the developmental cycle of $1.25 \%$ species and the estimation or observation of the lifespan of $0.45 \%$ species are presently known:

- known oribatid species

- number of species with descriptions of juvenile instars

- number of species with studies of life cycle duration

- number of species with studies / estimation of life span

$$
\begin{gathered}
\sim 11.500 \text { (173 families) } \\
898 \text { (120 families) } \\
144 \text { (42 families) } \\
52 \text { ( } 25 \text { families })
\end{gathered}
$$


The typical lifespan of an oribatid mite lasts one or two years in temperate to boreal regions (Norton and Behan-Pelletier 2009), rarely three years. In laboratory cultures the observed lifespan is generally shorter and decreases at higher temperatures. Very short longevities of adults were found in a nutritional experiment with some tropical species (Badejo \& Akinwole 2007) and in different relative humidities at different constant temperatures (Madge 1964). In Archegozetes longisetosus, also a tropical species, the short lifespan is influenced by stressful laboratory conditions or exposure to toxic substances (Seniczak 1998, 2006; Seniczak et al. 1999, 2005, 2006). Other tropical species show considerably faster development, and generally shorter lifespans, some species show lifespans of up to two years in the laboratory (Shereef 1976b). No field studies on the lifespan of oribatid mites have been carried out in the tropics. Long lifespan periods are particularly characteristic of species in polar regions (Alaskozetes antarcticus - Convey 1994; Ameronothrus lineatus-Søvik \& Leinaas 2003a; Maudheimia wilsoni-Marshall \& Convey 1999), but also in mountainous temperate regions (Canada: Ceratozetes gracilis, C. kananaskis — both estimates-Mitchell 1977b; Japan: Trhypochthoniellus brevisetus Kuriki 1995; European Alps: Oromurcia sudetica-Schatz 1985). The duration of the life cycle of a certain species can change in different geographic regions, as can be observed in Nothrus palustris (Grishina 1997) or in Ameronothrus lineatus (see below).

\section{Discussion}

The longevity of an oribatid mite depends on several environmental abiotic and biotic factors, such as temperature, light, humidity, soil acidity, duration of growing season, disturbances, availability and quality of food, or presence of toxic substances as heavy metals (e.g., Madge 1964; Siepel 1994; Maraun \& Scheu 2000; Ermilov \& Łochyńska 2008). It was recognized earlier on that temperature is likely to be one of the main parameters for the longevity of an oribatid species. At higher temperatures the developmental time tends to decrease (summarized in Lebrun 1970a; Luxton 1981a). Seniczak (2006) demonstrated in laboratory experiments on Archegozetes longisetosus that a high population density decreases the fertility of females, prolongs the developmental period of juveniles and increases the juvenile mortality, but has no influence on the lifespan of adults. In adults of Achipteria holomonensis high densities in culture also diminish the fecundity of females, while - in opposite to the previously mentioned species - the juvenile mortality and their developmental duration does not seem to be influenced (Stamou et al. 1981). As some authors suggest, the longevity might be shorter in more derived groups, while the dependence of the longevity on the animal size is controversial (e.g., Lebrun 1970a; Luxton 1981a; Haq \& Adolph 1981; Grishina 1991; Convey 1996). Siepel (1994) assumed that the genetic predisposition has a stronger influence on the generation time than other factors. However, heritable genetic variation of life-history traits is currently indistinguishable from phenotypic responsiveness to environmental conditions (Norton 1994). Molecular studies could provide a possible answer here.

Most of the data on lifespan of oribatid species are based on laboratory studies and can only be indicative of the potential natural lifespan. Adult oribatid mites survive frequently for a considerable period in culture. A comparison with field data was carried out rarely, mostly for species living under extreme conditions (e.g., Asikidis \& Stamou 1992; Schatz 1985; Wallwork et al. 1986; Convey 1994; Søvik \& Leinaas 2003a, b). Developmental times in culture and in the field can vary considerably. Luxton (1981a, b) studied the life cycle of several oribatid species in a temperate forest in Denmark where the majority of the species display an annual or two years cycle, in opposite to observations of some of the same species in the laboratory with much shorter developmental periods (e.g., Woodring \& Cook 1962, Luxton 1981a). These differences could be explained by long resting stages before moulting and/or the cold season of the year (Norton 1994), the latter factor can be diminished in semi-natural conditions in culture, e.g. in microcosms (e.g., Weigmann 1975; Schatz 1985; Convey 1994; Søvik \& Leinaas 2003a, b). The number of generations a species can have in a year or in another specified period of time will not be discussed here because it is beyond the focus of this survey, but this parameter was used several times for the estimation of developmental time from the field data (e.g., Weigmann 1975; Webb 1977; Thomas 1979; Haq \& Adolph 1981; Luxton 1981a; Haq \& Ramani 1984; Asikidis \& Stamou 1992). 
Few studies are available regarding the natural survival rate of each instar, although mortality is concentrated in immatures. During the pre-ecdysial resting stage and moulting oribatid mites are particularly vulnerable, as they are exposed to predators and can experience difficulties emerging from the exuvium (Lebrun \& van Straalen 1995). The mortality seems to decrease from egg to tritonymph (Schatz 1983; Kuriki 1995), but the survival probability of an egg reaching the adult instar can vary widely (e.g., Schatz 1985; Fernandez \& Athias Binche 1986; Mitchell 1977b; Convey 1994). Generally, older immature stages have longer developmental time (Luxton 1981a; Kaneko 1988b). In temperate and colder climate zones the life cycle is frequently extended, whereby different juvenile instars and adults can hibernate in dormancy (quiescence or diapause) thus reducing mortality (Belozerov 2008). An important survival tool during prolonged exposure to low temperatures without freezing is supercooling by synthesis of cryoprotectant substances. This phenomenon is known from numerous oribatid mites in cold climates, but also in temperate zones, especially in some alpine species (Sømme 1982; 1989; Cannon \& Block 1988). On the other hand, oribatid mites in hot deserts or in the Mediterranean climate with dry summer have evolved another kind of opportunistic adaptation, developing unique behaviour and exceptional life cycles by synchronizing the reproduction with the precipitation cycle (Wallwork et al. 1986; Stamou 1995). The hardened cuticle of oribatid mites (Norton 1994), the ptychoid defense mechanism (Schmelzle et al. 2015) and other defensive strategies, are adaptations of adults against predators, which may allow higher survival rates and thus longer lives.

\section{Some striking examples}

Alaskozetes antarcticus (Michael, 1903) (Fam. Podacaridae)

This species is widely distributed on sub-Antarctic islands and throughout the Antarctic Peninsula as a dominant member of many terrestrial communities in the maritime Antarctic (Block \& Convey 1995), and also found in some places in New Zealand (Luxton 1990). A detailed study of the life cycle of this species in the field and under seminatural conditions (field enclosures, $2-12^{\circ} \mathrm{C}$ ) has been carried out by Convey (1994). The life cycle of this species is not seasonal and all life stages overwinter in the field. Individual mites usually spend one year between moults, so the life cycle (from egg to egg) will last around 5 years. Adults survive at least two summers, females commence oviposition in their second summer, which can prolong the total lifespan to up to 7 years. This extended life cycle is exceptional and a special adaptation to survive the harsh environmental conditions of the Antarctic, linked with cold tolerance strategies such as increased resistance to desiccation by supercooling (Block \& Convey 1995).

\section{Ameronothrus spp. (Fam. Ameronothridae)}

The known species of this genus are bisexual, univoltine and larviparous, and occur mainly on marine coasts, in estuaries, but also in freshwater, saltmarshes and even in terrestrial habitats (Pfingstl 2017). Ameronothrus lineatus (Thorell, 1871) lives on coasts of the Canadian and Alaskan Arctic, Greenland, Svalbard, Scandinavia, also in temperate zones on the coasts of Germany, Netherlands, and the British Isles. Studies on development, life cycle and survival in the Arctic (Svalbard) were carried out by Søvik and Leinaas (2003a; b), Søvik et al. (2003), Søvik (2004) in laboratory microcosms under different temperature regimes and in the field. The development is interrupted by quiescence during the long polar winter. Females start larvipositing during the second adult summer, which gives a generation time from larva-to-larva of ca. 5 years. Consistent with the field data, the longevity of the adults is high, the longest-lived females survived almost five summers, while the majority of adults lived for 2-3 years. The total lifespan of A. lineatus in the Arctic can thus amount to 5-8 years.

The adult longevity was reduced at higher temperatures in laboratory experiments (Søvik \& Leinaas 2003b). This coincides with observations on A. lineatus in the temperate zone (Weser estuary, Germany, Bücking et al. 1998), where populations had a one-year life cycle. The seasonal development from larva to adult lasted 5-9 months, and most adults lived less than a year, dying in autumn after hatching of larvae.

Observations on other Ameronothrus species in the temperate zone show similar developmental times but longer periods of lifespan. Ameronothrus marinus (Banks, 1896) and A. maculatus (Michael, 1882), also in the Weser estuary, Germany (Bücking et al., 1998), had a developmental time in the field of about one year 
and a total longevity of 2 years. Ameronothrus schneideri (Oudemans, 1905) from the North Sea coast of Germany had a developmental cycle in the laboratory of about 11 months, the longevity of adults is unknown (Weigmann 1975). On the other hand, the lifespan of Ameronothrus lapponicus Dalenius, 1963 in the harsh conditions of Hardangervidda (Finse, South Norway) with extreme changes in temperature and long periods of severe drought is assumed to be 4-6 years (Tilrem 1994 in Bücking et al. 1998).

Archegozetes longisetosus Aoki, 1965 (Fam. Trhypochthoniidae)

This parthenogenetic species occurs in many tropical countries in the Oriental, Australian and Neotropical regions. Due to its relatively large size, short generation time, high fecundity and ease of culture Archegozetes longisetosus is among the most studied soil microarthropods under laboratory conditions, and certainly the best investigated oribatid mite species (Smrž \& Norton 2004; Heethoff et al. 2013). Numerous studies on its life history have been conducted, mainly regarding food preference and duration of development, some with different results (e.g., Haq \& Adolph 1981; Honciuc 1996; Seniczak 1998; Estrada-Venegas et al. 1999; Senizcak \& Senizcak 2002; Bergmann 2006; Heethoff et al. 2007; Seniczak et al. 2016; Brückner et al. 2018). The reason for this divergence could be attributed to the different origins of the animals, from India (Haq \& Adolph 1981), Mexico (Estrada-Venegas et al. 1999), and Puerto Rico (all other studies, based on a single gravid female, Bergmann 2006). Some studies show the influence of heavy metals (lead, cadmium, copper, zinc) on this species, prolonging the time of development, increasing mortality or reducing female fecundity (Seniczak et al. 1999, 2005, 2006, 2009, Seniczak \& Seniczak 2002). Heethoff et al. (2007), Seniczak et al. (2016) and Brückner et al. (2018) give following values from various laboratory cultures and food offers, based on own experiments and literature: the development time from egg to adult $28-88$ days and the longevity of adults 48-64 days. The total lifespan of A. longisetosus in culture is therefore 76-152 days, depending on temperature, possibly on the quality of food, presence or absence of heavy metals, density and origin of the specimens.

Oromurcia sudetica Willmann, 1939 (Fam. Ceratozetidae)

This species occurs in montane habitats in the Alps and Southeastern European mountains, also in the Caucasus. Subías (unpublished update 2007) treated this name as synonym of O. bicuspidata Thor, 1930 without any commentary. If this suggestion is valid, the species has a boreo-alpine distribution. The life cycle and the survival rate of this species in the Austrian Alps has been studied by Schatz $(1983 ; 1985)$ in laboratory and field studies. Individuals were kept under semi-natural conditions under temperature and light conditions corresponding to the multi-year average annual regime in the investigation area, a meadow above the timberline at $\sim 2000 \mathrm{~m}$ a.s.1. The mortality decreases with increasing age from egg to tritonymph. The life expectancy increases slightly from egg to larva, decreasing during the subsequent instars. The survival rate of adults is higher in winter than in summer. Overwintering specimens are inactive, and supercooling was proven (Schatz \& Sømme 1981; Sømme 1989). The life cycle is prolonged; breeding and hatching activity occurs only during the short vegetation period. The development from egg to adult takes a minimum of 2 years but can last up to 4 years. The observed longevity of the adults is one to two years. Thus, the total lifespan of Oromurcia sudetica in the investigated area can last between 3 and 6 years.

Considering the estimation of mortality rate with an equal gender ratio, each female has to lay 20 eggs in order to maintain the population density of Oromurcia sudetica constant in nature. This can be achieved through the long lifespan of the females and iteroparity (Schatz 1983).

\section{Conclusions}

Despite their high diversity, comparatively little is known about the longevity of most oribatid species. Several laboratory studies examined the developmental time under different conditions, but the lifespan of most oribatid mite species is unknown. The available results (less than a half percent of all known species) are mostly derived from laboratory observations. The few extrapolations on the real lifespan in the field originate from abundance studies and estimate the potential lifespan. The observed lifespans range from a few months to 8 years. The extreme longevities are known from polar regions, associated with rapid development in the growing seasons 
and long resting periods during the cold seasons, and are an excellent adaptation to the climatic conditions, demonstrating the climate as the most influential factor. Tropical species show generally faster development and shorter lifespans to species from temperate regions. Other factors, such as food quality or toxic substances, can also affect the lifespan of oribatid mites.

In view of the important role of oribatid mites as soil decomposers, knowledge about their life history traits and influencing parameters is essential, especially in the context of global climate change and increasing environmental pollution. With few exceptions, the studies on lifespans of oribatid mites were performed in the last decades of the previous century, most in the course of large-scaled ecological programs. At present, such long-term projects are difficult to obtain and finance, although the advanced technological tools would enable a study of evolutionary causes of lifespan at deeper levels by using new molecular biological methods.

\section{Acknowledgments}

We are grateful to Roy A. Norton who encouraged us to put this work together and provided important advice.

\section{References}

Adolph, C. \& Haq, M.A. (1984) Biology of Pelokylla malabarica Adolph \& Haq, 1982 (Acari: Oribatida). Acarology, 6 (2), 847-854.

Asikidis, M.D. \& Stamou, G.P. (1992) Phenological patterns of oribatid mites in an evergreen-sclerophyllous formation (Hortiatis, Greece). Pedobiologia, 36, 359-372.

Badejo, M.A. \& Akinwole, P.O. (2007) Preliminary study of the feeding habitats of seven species of oribatid mites from Nigeria. Systematic \& Applied Acarology, 12 (2), 121-125. https://doi.org/10.11158/saa.12.2.5

Bale, J.S., Hodkinson, I.D., Block, W., Webb, N.R., Coulson, S.C. \& Strathdee, A.T. (1997) Life strategies of arctic terrestrial arthropods. In: Woodin, S.J. \& Marquiss, M. (Eds.), Ecology of Arctic Environments. Special Publication of The British Ecological Society, Nr. 13, pp. 137-165.

Bäumler, W. (1970) Zur Morphologie, Biologie und Ökologie von Hermannia gibba (Acarina, Oribatei) unter Berücksichtigung einiger Begleitarten. Zeitschrift für angewandte Entomologie, 66, 337-362.

Behan-Pelletier, V. \& Eamer, B. (2007) Aquatic Oribatida: adaptations, constraints, distribution and ecology. In: MoralesMalacara, J.B., Behan-Pelletier, V., Ueckermann, E., Pérez, T.M., Estrada-Venegas, E.G. \& Badil, M. (Eds.), Acarology XI: Proceedings of the International Congress. Instituto de Biología and Facultad de Ciencias, Universidad Nacional Autónoma de México; Sociedad Latinoamericana de Acarología, México, pp. 71-82.

Bellido, A. (1979) Écologie de Carabodes willmanni Bernini 1975 (Acari, Oribatei) dans les formations pionièrres de la lande armoricaine. Revue d'Écologie et de Biologie du Sol, 16, 195-218.

Belozerov, V.N. (2008) Diapause and quiescence as two main kinds of dormancy and their significance in life cycles of mites and ticks (Chelicerata: Arachnida: Acari). Part 1. Acariformes. Acarina, 16 (2), 79-130.

Bergmann, P.K. (2006) Untersuchungen zur Reproduktionsbiologie der parthenogenetischen Hornmilbe Archegozetes longisetosus (Acari: Sarcoptiformes: Trhypochthoniidae). Diplomarbeit, Fakultät für Biologie der Eberhard-KarlsUniversität Tübingen, 104 pp.

Bhattacharya, T., Joy, V.C. \& Joy, S. (1978) Studies on the effect of temperature on the development of Oppia nodosa Hammer (Acari: Cryptostigmata: Oppiidae). Entomon, 3 (2), 149-155.

Block, W. (1965) The life histories of Platynothrus peltifer (Koch 1836) and Damaeus clavipes (Hermann 1804) (Acari Oribatei) in soils of pennine moorland. Acarologia, 7 (4), 735-743.

Block, W. \& Convey, P. (1995) The biology, life-cycle and ecophysiology of the Antarctic mite Alaskozetes antarcticus. Journal of Zoology, London, 236 (3), 431-449. https://doi.org/10.1111/j.1469-7998.1995.tb02723.x

Brückner, A., Schuster, R., Wehner, K. \& Heethoff, M. (2018) Effects of nutritional quality on the reproductive biology of Archegozetes longisetosus (Actinotrichida, Oribatida, Trhypochthoniidae). Soil Organisms, 90 (1), 1-12.

Bücking, J., Ernst, H. \& Siemer, F. (1998) Population dynamics of phytophagous mites inhabiting rocky shores - K-strategists in an extreme environment? In: Ebermann, E. (Ed.), Arthropod Biology: Contributions to Morphology, Ecology and Systematics. Biosystematics and Ecology Series, 14, pp. 93-144.

Cannon, R.S.C. \& Block, W. (1988) Cold tolerance of microarthropods. Biological Reviews, 63 (1), $23-77$. https://doi.org/10.1111/j.1469-185X.1988.tb00468.x

Cleat, N.D. (1952) Growth in the laboratory of economically important oribatid mites. Nature, 169, 280-281. 
https://doi.org/10.1038/169280b0

Convey, P. (1994) Growth and survival strategy of the Antarctic mite Alaskozetes antarcticus. Ecography, 17 (1), $97-107$. https://doi.org/10.1111/j.1600-0587.1994.tb00081.x

Convey, P. (1996) Overwintering strategies of terrestrial invertebrates in Antarctica - the significance of flexibility in extremely seasonal environments. European Journal of Entomology, 93, 489-505.

Cordo, H.A. \& DeLoach C.J. (1975) Ovipositional specificity and feeding habits of the Waterhyacinth Mite, Orthogalumna terebrantis, in Argentina. Environmental Entomology, 4 (4), 561-565. https://doi.org/10.1093/ee/4.4.561

Denmark, M.A. \& Woodring, G.P. (1965) Feeding habits of Hemileius new species (Acari: Cryptostigmata, Oribatulidae) on Florida orchids. The Florida Entomologist, 48 (1), 9-16. https://doi.org/10.2307/3493516

Elmoghazy, M.M.E., El-Kawas, H.M.G. \& Salman, M.S. (2012) Biological aspects of Scheloribates laevigatus (Acari: Oribatida) when fed on mixture of the free-living nematod, Eudiplogaster phlagellicaudatus and potato in the laboratory. Acarines, $6,21-24$. https://doi.org/10.21608/ajesa.2012.163620

Enami, Y. (1992) Life history of Epidamaeus verrucatus Enami et Fujikawa (Acari: Damaeidae), with morphological description of its immature stage. Edaphologia, 48, 23-29.

Ermilov, S.G. (2006) The life cycle of Hydrozetes lemnae (Oribatei, Hydrozetidae). Entomological Review, 86 (Suppl. 2), $177-181$. https://doi.org/10.1134/S0013873806110108

Ermilov, S.G. (2007) The postembryonic development of Camisia biurus (Oribatei, Camisiidae). Entomological Review, 87 (2), 222-230. https://doi.org/10.1134/S0013873807020091

Ermilov, S.G. (2008) The laboratory cultivation of oribatid mites of the superfamily Crotonioidea (Acari, Oribatida) with the purpose of studying their development. Ministry of Agriculture of the Russian Federation, Nizhniy Novgorod, 54 pp. [in Russian]

Ermilov, S.G., Chistyakov, M.P. \& Renzhina, A.A. (2004) The effect of temperature on the duration of development of Trhypochthonius tectorum (Berlese, 1896) (Acariformes, Oribatei). Povolzhskiy Ecologicheskiy Zhurnal, 1, 87-90. [in Russian]

Ermilov, S.G. \& Łochyńska, M. (2008) The influence of temperature on the development time of three oribatid mite species (Acari, Oribatida). North-Western Journal of Zoology, 4 (2), 274-281.

Ermilov, S.G., Łochyńska, M. \& Olszanowski, Z. (2008) The cultivation and morphology of juvenile stages of two species from the genus Scutovertex (Acari: Oribatida: Scutoverticidae). Annales Zoologici, 58 (2), 433-443. https://doi.org/10.3161/000345408X326762

Estrada-Venegas, E., Norton, R.A., Equihuq-Martinez, J., Poles, N., Trinidad, S.J. \& Gonzalez, H.J. (1999) Biología y nueva sinonimia de Archegozetes longisetosus Aoki (Acari-Oribatida) de la Ma.

Fernandez, N.A. \& Athias-Binche, F. (1986) Analyse démographique d'une population d'Hydrozetes lemnae Coggi, Acarien Oribate inféodé a la lentille d'eau Lemna gibba L. en Argentine. I. Méthodes et techniques, démographie d' H. lemnae comparaisons avec d'autres Oribates. Zoologisches Jahrbuch Systematik, 113 (2), 213-228.

Gourbière, F., Lions, J.C. \& Pepin, R. (1985) Activité et développement d'Adoristes ovatus (C.L. Koch, 1839) (Acarien, Oribate) dans les aiguilles d'Abies alba Mill. Relations avec la décomposition et les microflores fongiques. Revue d'écologie et de biologie du sol, 22 (1), 57-73.

Grandjean, F. (1950) Observations ethologiques sur Camisia segnis (Herm.) et Platynothrus peltifer (Koch) (Acariens). Bulletin du Muséum National d'Histoire Naturelle, (2), 22, 224-231.

Grishina, L.G. (1991) The duration of oribatids (Sarcoptiformes, Oribatei) life cycle. Siberian Biological Journal, 1991 (3), 38-47. [in Russian]

Grishina, L.G. (1997) Population dynamics of oribatid mites in different parts of species areas. Abhandlungen und Berichte des Naturkundemuseums Görlitz, 69 (6), 53-56.

Haarløv, N. (1960) Microarthropods from Danish soils. Ecology, Phenology. Oikos, 3, 1-176.

Hågvar, S. (1998) Mites (Acari) developing inside decomposing spruce needles: Biology and effect on decomposition rate. Pedobiologia, 42, 358-377.

Haq, M.A. \& Adolph, C. (1981) A comparative study of the duration of the life cycles of 4 species of oribatid mites (Acari Oribatei) from the soils of Kerala, India. Indian Journal of Acarology, 5 (1-2), 56-61.

Haq, M.A. \& Ramani, N. (1984) Postembryonic development of Paralamellobates bengalensis Bhaduri \& Chaudhuri 1968 (Acari: Oribatei) parasitic on Dioscorea alata. In: Griffiths, D.A. \& Bowman, C.E. (Eds.), Acarology VI, Vol. 2. Horwood, Chichester, pp. 819-825.

Haq, M.A. \& Shereef, E.A.M. (1992) Post-embryonic development of a species of Galumna von Heyden (Acari: Oribatei). In: Haq, M.A. \& Ramani, N. (Eds.), Man, mites and environment. Anjengo Publications, Calicut University, P.O., Kerala, pp. $155-160$.

Haq, M.A., Mubarak, K.V.A. \& Ramani, N. (1984) New lohmanniid mites (Acari: Oribatei) from Kerala, India. Indian Journal of Acarology, 8, 87-94. 
Harding, D.J.L. (1971) The phenology of Platynothrus peltifer (C.L. Koch). In: Daniel, M. \& Rosicky, B. (Eds.), Proceedings of the 3rd International Congress of Acarology, Czechoslovak Academy of Sciences, Prague, pp. 79-83. https://doi.org/10.1007/978-94-010-2709-0_13

Hartenstein, R. (1962a) Soil Oribatei II. Belba kingi, new species (Acarina: Belbidae), and a study of its life history. Annals of the Entomological Society of America, 55 (4), 357-361. https://doi.org/10.1093/aesa/55.4.357

Hartenstein, R. (1962b) Soil Oribatei III. Studies on the development, biology, and ecology of Metabelba montana (Kulcz.) (Acarina: Belbidae) and Eremobelba nervosa n. sp. (Acarina: Eremaeidae). Annals of the Entomological Society of America, 55 (4), 361-367. https://doi.org/10.1093/aesa/55.4.361

Hartenstein, R. (1962c) Soil Oribatei IV. Observations on Ceratozetes gracilis (Acarina: Ceratozetidae). Annals of the Entomological Society of America, 55 (5), 583-586. https://doi.org/10.1093/aesa/55.5.583

Hartenstein, R. (1962d) Soil Oribatei V. Investigations on Platynothrus peltifer (Acarina: Camisiidae). Annals of the Entomological Society of America, 55 (6), 709-713. https://doi.org/10.1093/aesa/55.6.709

Hartenstein, R. (1962e) Soil Oribatei VI. Protoribates lophotrichus (Acarina: Haplozetidae) and its associations with microorganisms. Annals of the Entomological Society of America, 55 (5), 587-591. https://doi.org/10.1093/aesa/55.5.587

Heba, M.F. (2019) Effect of different soil fungi on biological aspects of the oribatid mite Nothrus silvestris (Acari: Oribatida) in the laboratory. Egyptian Journal of Plant Protection Research Institute, 2 (1), 81-87.

Heethoff, M., Bergmann, P., Laumann, M. \& Norton R.A. (2013) The 20th anniversary of a model mite: a review of current knowledge about Archegozetes longisetosus (Acari, Oribatida). Acarologia, 53 (4), 353-368. https://doi.org/10.1051/acarologia/20132108

Heethoff, M., Laumann, M. \& Bergmann, P. (2007) Adding to the reproductive biology of the parthenogenetic oribatid mite, Archegozetes longisetosus (Acari, Oribatida, Trhypochthoniidae). Turkish Journal of Zoology, 31, 151-159.

Honciuc, V. (1996) Laboratory studies of the behavior and life cycle of Archegozetes longisetosus Aoki 1965 (Oribatida). In: Mitchell, R., Horn, D.J., Needham, G.R. \& Welbourn, C.W. (Eds.), Acarology IX-Proceedings. Ohio Biological Survey, Columbus, Ohio, vol. 1, pp. 637-640.

Jalil, M. (1965) The life cycle of Hermannia scabra (c.L. Koch, 1879) (Acarina-Oribatei). Oikos, 16, 16-19. https://doi.org/10.2307/3564860

Jalil, M. (1969) The life cycle of Humerobates rostrolamellatus Grandjean, 1936 (Acari). Journal of the Kansas Entomological Society, 42, 526-530.

Jalil, M. (1972) A note on the life cycle of Platynothrus peltifer. Journal of the Kansas Entomological Society, 45, 309-311.

Kaneko, N. (1988a) Life history of Oppiella nova (Oudemans) (Oribatei) in cool temperate forest soils in Japan. Acarologia, 29 (2), 215-221.

Kaneko, N. (1988b) A review of life history studies on oribatid mites. Edaphologia, 39, 1-9.

Kaneko, N. (1989) Life histories of four oribatid mite species in a mull type soil in a cool temperate forest in Japan. Pedobiologia, 33, 117-126.

Koch, C.L. (1835) Deutschlands Crustaceen, Myriapoden und Arachniden, Heft 3. F. Pustet, Regensburg.

Koch, C.L. (1839) Deutschlands Crustaceen, Myriapoden und Arachniden, Heft 29. F. Pustet, Regensburg.

Koch, C.L. (1841) Deutschlands Crustaceen, Myriapoden und Arachniden, Heft 31. F. Pustet, Regensburg.

Kuriki, G. (1995) Life cycle of Trhypochthoniellus setosus Willmann (Acari: Trhypochthoniidae) in a Sphagnum moor at Yachidaira, northeast Japan. Journal of the Acarological Society of Japan, 4 (2) 113-122. https://doi.org/10.2300/acari.4.113

Kuriki, G. (2008) The life cycle of Limnozetes ciliatus (Schrank, 1803) (Acari: Oribatida). Journal of the Acarological Society Japan, 17 (2), 75-85. https://doi.org/10.2300/acari.17.75

Kuriki, G. (2010) The life cycle of Trhypochthoniellus crassus (Warburton et Pearce, 1905) (Acari: Oribatida). Edaphologia, $87,49-56$. https://doi.org/10.20695/edaphologia.87.0_49

Lebrun, P. (1970a) Écologie et biologie de Nothrus palustris (C.L. Koch, 1839). $3^{\mathrm{e}}$ note. Cycle de vie. Acarologia, 12 (1), 193-207.

Lebrun, P. (1970b) Écologie et biologie de Nothrus palustris (C.L. Koch, 1839) - Acarien - Oribate. IV. Survivance - Fecondite - Action d'un predateur. Acarologia, 12 (4), 827-848.

Lebrun, P. (1971) Écologie et biocénotique de quelques peuplements d'arthropodes édaphiques. Mémoires de l'Institut Royal des Sciences Naturelles de Belgique, 165, 1-203.

Lebrun, P.H. (1974) Écologie du développement de Damaeus onustus et Damaeus clavipes (Acariens, Oribates) influence de la température. Acarologia, 16 (2), 344-357.

Lebrun, P., van Impe, G., De Saint Georges Gridelet, D., Wauthy, G. \& André, H.M. (1991) The life strategies of mites. In: Schuster, R. \& Murphy, P.W. (Eds.), The Acari-Reproduction, development and life-history strategies. Chapman and Hall, 
London - New York, pp. 3-22.

https://doi.org/10.1007/978-94-011-3102-5_1

Lebrun, P. \& van Straalen, N.M. (1995) Oribatid mites: prospects of their use in ecotoxicology. Experimental and Applied Acarology, 19 (7), 361-380. https://doi.org/10.1007/BF00145154

Luxton, M. (1981a) Studies on the oribatid mites of a Danish beech wood soil. IV. Developmental biology. Pedobiologia, 21, $312-340$.

Luxton, M. (1981b) Studies on the oribatid mites of a Danish beech wood soil. VI. Seasonal population changes. Pedobiologia, 21, 387-409.

Luxton, M. (1990) The marine littoral mites of the New Zealand region. Journal of the Royal Society of New Zealand, 20 (4), 367-418. https://doi.org/10.1080/03036758.1990.10426719

Madge, D.S. (1964) The longevity of fasting oribatid mites. Acarologia, 6 (4), 718-729.

Maraun, M. \& Scheu, S. (2000) The structure of oribatid mite communities (Acari, Oribatida): patterns, mechanisms and implications for future research. Ecography, 23 (3), 374-383. https://doi.org/10.1111/j.1600-0587.2000.tb00294.x

Marshall, D.J. \& Convey, P. (1999) Compact aggregation and life history strategy in a continental Antarctic mite. In: Bruin, J., van der Geest, L.P.S. \& Sabelis, M. (Eds.), Ecology and evolution of the Acari. Kluwer Academic Publishers, Dordrecht, The Netherlands, pp. 557-567. https://doi.org/10.1007/978-94-017-1343-6_47

Michael, A.D. (1880) Further contribution to the knowledge of British Oribatidae. (Part I). Royal Microscopical Society, 3 , 32-43.

Michael, A.D. (1884) British Oribatidae. Vol. I. Ray Society, London, pp. 1-336.

Mitchell, M.J. (1977a) Life history strategies of oribatid mites. In: Dindal, D.L. (Ed.), Biology of Oribatid Mites. SUNY College of Environmental Science and Forestry, Syracuse, pp. 65-69.

Mitchell, M.J. (1977b) Population dynamics of oribatid mites (Acari: Cryptostigmata) in an aspen woodland soil. Pedobiologia, 17, 305-319.

Murphy, P.W. \& Jalil, M. (1964) Some observations on the genus Tectocepheus. Acarologia, fascicle hour series, $187-197$ (C. R. ler Congres Int. d'Acarologie, Fort Collins, Col., U.S.A., 1963).

Nannelli, R. (1975) Osservazioni sulla biologia di Oppia concolor Koch (Acarina, Oribatei, Oppiidae) in condizioni sperimentali di allevamento. Redia, 56, 111-116.

Narsapur, V.S. (1983) Studies on the biology of Scheloribates laevigatus (Acari: Oribatei) in India. Indian Journal of Acarology, $8(1), 44-48$.

Nicolet, H. (1855) Histoire naturelle des Acariens qui se trouvent aux environs de Paris. Archives du Muséum d'Histoire Naturelle, Paris, 7, 381-482. https://doi.org/10.5962/bhl.title.66066

Norton, R.A. (1994) Evolutionary aspects of oribatid mite life histories and consequences for the origin of the Astigmata. In: Houck, M. (Ed.), Mites. Ecological and evolutionary analyses of life-history patterns. Chapman and Hall, New York, pp. 99-135. https://doi.org/10.1007/978-1-4615-2389-5_5

Norton, R.A. (1998) Morphological evidence for the evolutionary origin of Astigmata (Acari: Acariformes). Experimental \& Applied Acarology, 22, 559-594. https://doi.org/10.1023/A:1006135509248

Norton, R.A. \& Palmer, S.C. (1991) The distribution, mechanisms and evolutionary significance of parthenogenesis in oribatid mites. In: Schuster, R. \& Murphy, P.W. (Eds.), The Acari-Reproduction, development and life-history strategies. Chapman and Hall, London - New York, pp. 107-136. https://doi.org/10.1007/978-94-011-3102-5_7

Norton, R.A. \& Behan-Pelletier, V. (2009) Chapter 15, Oribatida. In: Krantz, G.W. \& Walter, D.E. (Eds.), A Manual of Acarology 3rd Edition. Texas Tech. University Press, Lubbock, pp. 421-564.

Norton, R.A. \& Ermilov, S.G. (2014) Catalogue and historical overview of juvenile instars of oribatid mites (Acari: Oribatida). Zootaxa, 3833, 1-132. [with corrections emendations November 2021] https://doi.org/10.11646/zootaxa.3833.1.1

Norton, R.A., Kethley, J.B., Johnston, D.E. \& OConnor, B.M. (1993) Phylogenetic perspectives on genetic systems and reproductive modes of mites. In: Wrensch, D.L. \& Ebbert, M.A. (Eds.), Evolution and diversity of sex ratio in insects and mites. Chapman and Hall, New York, pp. 8-99. https://doi.org/10.1007/978-1-4684-1402-8_2

Paez, J., Villagomez, F. \& Palacios-Vargas, J.G. (2019) Description of a new Pergalumna (Acari: Oribatida: Galumnidae) species from Mexico and its postembryonic development. Zootaxa, 4647 (1) 385-406. https://doi.org/10.11646/zootaxa.4647.1.25

Pauly, F. (1956) Zur Biologie einiger Belbiden (Oribatei: Moosmilben) und zur Funktion ihrer pseudostigmatischen Organe. Zoologisches Jahrbuch Systematik, 84: 275-328. 
Pfingstl, T. (2017) The marine-associated lifestyle of ameronothroid mites (Acari, Oribatida) and its evolutionary origin: a review. Acarologia, 57 (3), 693-721. https://doi.org/10.24349/acarologia/20174197

Ramani, N. (1999) Biological studies on Xylobates seminudus (Acari: Oribatei), a potential species in biodegradation. Journal of Acarology, 14 (1\&2), 69-72.

Ramani, N. \& Haq, M.A. (1987) Temperature as a regulative factor on the development of Scheloribates decarinatus Aoki, 1984 (Acari: Oribatei). In: Ismail, A.S. \& Alawdeen, S.S. (Eds.), Aspects of behaviour, V.P.S. Printers, Madras, pp. 92-97.

Ramani, N. \& Haq, M.A. (1988) Developmental studies of Uracrobates indicus (Acari: Oribatei) inhabiting Mangifera indica. In: Channabasavanna, G.P. \& Viraktamath, C.A. (Eds.), Progress in Acarology, Vol. 1. Brill, Leiden, pp. 483-490.

Ramani, N. \& Haq, M.A. (1993) Influence of food on the development of Allonothrus giganticus Haq (Acari: Oribatei). In: Rajagopal, D. \& Kale, R.D. (Eds.), Soil Organisms and Sustainability. Proceedings IV Nat. Symp. Soil Biol. Ecol. ISSBE UAS, Bangalore, pp. 189-194.

Reddy, A.S., Kumar, R. \& Mathur, S.N. (1978) Studies on the Biology of Galumna flabellifera Hammer, 1958 and its role as fungal spore carrier. In: Edwards, C.A. \& Vereesh, G.K. (Eds.), Soil Biology and Ecology in India. Univ. Agri. Sci., Tech. Ser. 22. Hebbal, Bangalore, pp. 134-143.

Reeves, R.M. (1991) Carabodes niger Banks, C. polyporetes n.sp. and unverified records of C. areolatus Berlese (Acari, Oribatida, Carabodidae) in North America. Canadian Journal of Zoology, 69 (12), 2925-2934. https://doi.org/10.1139/z91-413

Rockett, C.L. \& Woodring, J.P. (1966) Biological investigations of a new species of Ceratozetes and of Pergalumna (Acarina: Cryptostigmata). Acarologia, 8, 511-520.

Rohde, C.J. (1955) Studies on Arthropods from a moss habitat with special emphasis on the life history of three oribatid mites. Dissertation Abs. 15: 1936 Northwestern University, Illinois.

Saichue, P., Gerson, U. \& Henis, Y. (1972) Observations on the feeding and life history of the mite Nothrus biciliatus (Koch). Soil Biology and Biochemistry, 4, 155-164. https://doi.org/10.1016/0038-0717(72)90006-5

Schatz, H. (1983) Überlebensrate von Oromurcia sudetica Willmann (Acari, Oribatei) von einer alpinen Wiese Tirols (Obergurgl, Zentralalpen). Zoologisches Jahrbuch Systematik, 110, 97-109.

Schatz, H. (1985) The life cycle of an alpine oribatid mite, Oromurcia sudetica Willmann. Acarologia, 26 (1), 95-100.

Schatz, H. \& Sømme, L. (1981) Cold-hardiness of some oribatid mites from the Alps. Cryo-Letters, 2, $207-216$.

Schatz H. \& Behan-Pelletier V.M. (2008) Global diversity of oribatids (Oribatida; Acari - Arachnida). In: Balian, E.V., Lévêque, C., Segers, H. \& Martens, K. (Eds.), Freshwater Animal Diversity Assessment. Hydrobiologia, 595 pp. 323-328. https://doi.org/10.1007/978-1-4020-8259-7_35

Schenker, R. (1987) Biological and ecological notes on the mite Epidamaeus diversipilis. In: Striganova, B.R. (Ed.), Soil fauna and soil fertility. Proceedings of the $9^{\text {th }}$ international colloquium on soil zoology. Nauka, Moscow, pp. 581-583.

Schmelzle, S., Norton, R.A. \& Heethoff, M. (2015) Mechanics of the ptychoid defense mechanism in Ptyctima (Acari, Oribatida): One problem, two solutions. Zoologischer Anzeiger, 254, 27-40. https://doi.org/10.1016/j.jcz.2014.09.002

Sengbusch, H.G. (1954) Studies on the life history of three oribatoid mites with observations on other species. Annals of the Entomological Society of America, 47 (4), 646-667. https://doi.org/10.1093/aesa/47.4.646

Sengbusch, H.G. (1958a) The development of Nanhermannia nana (Nicolet), (Acarina, Oribatei); life history studies of Oribatei II. The Anatomical Record, 132 (3), 504.

Sengbusch, H.G. (1958b) Zuchtversuche mit Oribatiden (Acarina). Naturwissenschaften, 20, 498-499. https://doi.org/10.1007/BF00635575

Sengbusch, H.G. \& Sengbusch, C.H. (1970) Post-embryonic development of Oppia nitens (Acarina: Oribatei). Journal of the New York Entomological Society, 78 (4), 207-214.

Seniczak, A. (1998) Preliminary studies on the influence of food on the development and morphology of Archegozetes longisetosus Aoki (Acari: Oribatida) in laboratory conditions. Akdemia Techniczno-Rolnicza Im. Jana i Jedrzeja Sniadeckich w Bydgoszczy. Zeszyty Naukowe Nr. 214, Ochrona Srodowiska, 2, 175-180.

Seniczak, A. (2006) The effect of density on life-history parameters and morphology of Archegozetes longisetosus Aoki, 1965 (Acari: Oribatida) in laboratory conditions. Biological Letters, 43 (2), 209-213.

Seniczak, A. (2007) Preliminary studies on the toxicity of copper and lead in Pergalumna nervosa (Berlese, 1914) (Acari, Oribatida) in laboratory tests. In: Tajovský, K., Schlaghamerský, J. \& Pižl, V. (Eds.), Contributions to Soil Zoology in Central Europe II. ISB BC AS CR, v.v.i., České Budějovice, pp. 131-134.

Seniczak, A. \& Seniczak, S. (2002) The effect of cadmium on Archegozetes longisetosus (Acari, Oribatida) in laboratory conditions. European Journal of Soil Biology, 38 (3-4), 315-317. https://doi.org/10.1016/S1164-5563(02)01166-4

Seniczak, A., Ligocka, A., Seniczak, S. \& Paluszak, Z. (2009) The influence of cadmium on life-history parameters and gut microflora of Archegozetes longisetosus (Acari, Oribatida) under laboratory conditions. Experimental and Applied Acarology, 47 (3), 191-200. https://doi.org/10.1007/s10493-008-9210-6 
Seniczak, A., Ligocka, A., Seniczak, S. \& Paluszak, Z. (2016) Effects of green algae and napa cabbage on life-history parameters and gut microflora of Archegozetes longisetosus (Acari: Oribatida) under laboratory conditions. Biological Letters, 53 (2), $67-78$. https://doi.org/10.1515/biolet-2017-0007

Seniczak, A., Seniczak, S. \& Długosz, J. (1999) The effect of lead and copper on Archegozetes longisetosus Aoki (Acari, Oribatida) in laboratory conditions. In: Tajovsky, K. \& Pizl, V. (Eds.), Soil Zoology in Central Europe. Institute of Soil Biology. Academy of Sciences, Ceské Budejovice, pp. 289-293.

Seniczak, A., Seniczak, S. \& Długosz, J. (2005) The effect of lead and zinc on the moss mite Archegozetes longisetosus Aoki (Acari, Oribatida) under laboratory conditions. In: Tajovský, K., Schlaghamerský, K. \& Pizl, V. (Eds.), Contributions to Soil Zoology in Central Europe I. Institute of Soil Biology. Academy of Sciences, Ceské Budejovice, pp. 133-136.

Seniczak, A., Seniczak, S. \& Kobierski, M. (2006) Long-term effect of cadmium on the oribatid mite Archegozetes longisetosus Aoki, 1965 in laboratory conditions. Biological Letters, 43 (2), 237-242.

Seniczak, A., Seniczak, S., Kaczmarek, S. \& Chachaj, B. (2017) Morphological ontogeny and ecology of Adoristes ovatus (Acari: Oribatida: Liacaridae), with comments on Adoristes Hull. Systematic \& Applied Acarology, 22 (12), $2038-2056$. https://doi.org/10.11158/saa.22.12.2

Shaldybina, E.S. (1984) Life cycle of Nothrus palustris (Oribatei, Nothroidea). Zoologicheskii Zhurnal, 63, 671-676. [in Russian]

Shereef, G.M. (1972) Observations on Oribatid mites in laboratory cultures. Acarologia, 14 (2), $282-291$.

Shereef, G.M. (1976a) Biology of two Oribatid species in Giza region. Acarologia, 18 (1), 171-173.

Shereef, G.M. (1976b) Biological studies and description of stages of two species. Papillacarus aciculatus Kunst and Lohmannia egypticus Elbadry and Nasr (Oribatei-Lohmanniidae) in Egypt. Acarologia, 18 (2), 352-359.

Shereef, G.M. (1977) Biological studies and description of developmental stages of Plakoribates multicuspidatus Popp. and Xylobates souchnaiensis Abdel-Hamid (Acarina, Oribatei) in Egypt. Acarologia, 18 (4), 748-753.

Shereef, G.M. \& Haq, M.A. (1992) Biology of Galumna triquetra Aoki, 1965, a vector mite of cestode parasites. In: Ramachandran Nair, C.G. (Ed.), Proceedings of the 4th Kerala Science Congress, Thrissur, pp. 238-240.

Shereef, G.M. \& Zaher, M.A. (1980) Morphological and biological studies on Oppia bayoumi sp. n. from Egypt (Cryptostigmata: Oppiidae). Bulletin - Zoological Society Egypt, 30, 7-14.

Siepel, H. (1994) Life-history tactics of soil microarthropods. Biology and Fertility of Soils, 18 (4), 263-278. https://doi.org/10.1007/BF00570628

Smrž, J. \& Norton, R.A. (2004) Food selection and internal processing in Archegozetes longisetosus (Acari: Oribatida). Pedobiologia, 48, 111-120. https://doi.org/10.1016/j.pedobi.2003.09.003

Sølhoy, T. (1975) Dynamics of Oribatei populations on Hardangervidda. In: Wielgolaski, F.E. (Ed.), Fennoscandian Tundra Ecosystems, Part 2. Ecological Studies. Analysis and Synthesis. Springer, Berlin-Heidelberg-New York, 17, pp. 60-65.

Sømme, L. (1982) Supercooling and winter survival in terrestrial arthropods. Comparative Biochemistry and Physiology, 73A (4), 519-543. https://doi.org/10.1016/0300-9629(82)90260-2

Sømme, L. (1989) Adaptations of terrestrial arthropods to the alpine environment. Biological Reviews, 64, 367-407. https://doi.org/10.1111/j.1469-185X.1989.tb00681.x

Søvik, G. \& Leinaas, H.P. (2003a) Long life cycle and high adult survival in an arctic population of the mite Ameronothrus lineatus (Acari, Oribatida) from Svalbard. Polar Biology, 26, 500-508. https://doi.org/10.1007/s00300-003-0510-3

Søvik, G. \& Leinaas, H.P. (2003b) Adult survival and reproduction in an arctic mite, Ameronothrus lineatus (Acari, Oribatida): Effects of temperature and winter cold. Canadian Journal of Zoology, 81, 1579-1588. https://doi.org/10.1139/z03-113

Søvik, G. (2004) The biology and life history of arctic populations of the littoral mite Ameronothrus lineatus (Acari, Oribatida). Experimental and Applied Acarology, 34 (1-2), 3-20. https://doi.org/10.1007/978-94-017-0429-8_2

Søvik, G., Leinaas, H.P., Ims, R.A. \& Solhøy, T. (2003) Population dynamics and life history of the oribatid mite Ameronothrus lineatus (Acari, Oribatida) on the high Arctic Archipelago of Svalbard. Pedobiologia, 47, 257-271. https://doi.org/10.1078/0031-4056-00189

Stamou, G.P. (1989) Studies on the effect of temperature on the demographic parameters of Achipteria holomonensis (Acari, Oribatida). Acarologia, 30 (2), 171-180.

Stamou, G.P. (1995) Strategic responses of oribatid mites to the severity of the mediterranean environment. In: Kropczynska, D., Boczek, J. \& Tomczyk, A. (Eds.), The Acari - Physiological and Ecological Aspects of Acari - Host Relationships. Oficyna Dabor, Warszawa, pp. 295-304.

Stamou, G.P., Kattoulas, M., Cancela da Fonseca, J.P. \& Margaris, N.S. (1981) Observations on the biology and ecology of Achipteria holomonensis (Acarina, Oribatida). Pedobiologia, 23, 53-58.

Stunkard, H.W. (1944) Studies on the life history of the oribatid mite Galumna sp., intermediate host of Moniezia expansa. The Anatomical Record 89, 550.

Subías, L.S. (2004 -2021) Listado sistemático, sinonímico y biogeográfico de los ácaros oribátidos (Acariformes, Oribatida) del 
mundo (excepto fósiles). Graellsia, 60 (número extraordinario), 3-305 (2004), actualized pdf 2007, 2021. Online capture. https://doi.org/10.3989/graellsia.2004.v60.iExtra.218

Syamjith, P.K. \& Ramani, N. (2020) Biology of the oribatid mite Acrotritia clavata (Märkel, 1964) from the mangrove ecosystems of North Kerala, India. Acarological Studies, 2 (2), 59-68. https://doi.org/10.47121/acarolstud.669572

Taberly, G. (1957) Observations sur les spermatophores et leur transfert chez les Oribates (Acariens). Bulletin de la Société zoologique de France, 82 (1), 140-145.

Taberly, G. (1987) Compléments sur le cycle de vie de Trhypochthonius tectorum (Berlese) (Acarien, Oribatei). Vie et Milieu, 37, 221-228.

Taberly, G. (1988) Cycle de vie de Platynothrus peltifer (Koch) (Acarien, Oribate). Vie et Milieu, 38 (1), 85-94.

Tanimoto, Y. (1980) The life history of Pergalumna sp. (Oribatei, Galumnidae). The Nanki Biological Society, Nankiseibutu, 22 (1), 26-28.

Thomas, J.O.M. (1979) An energy budget for a woodland population of oribatid mites. Pedobiologia, 19, 346-378.

Tilrem L. (1994) Life history traits in two oribatid mites (Ameronothrus lineatus Dalenius and Phauloppia sp.) in an extreme high mountain. Thesis candidata scientiarum, University of Bergen, Norway, $58 \mathrm{pp}$.

Travé, J. \& Duran, F. (1971) Développement et comportement en laboratoire de Neoribates gracilis Travé (Acarien, Oribate). Vie et Milieu, 22 (1), 79-89.

Travnicek, M. (1989) Laboratory cultivation and biology of mites in the family Liacaridae (Acari: Oribatida). Acta Universitatis Carolinensis, Praha, 33 (2), 69-80.

Wallwork, J.A. (1980) Desert soil microarthropods, an "r"-selected system. In: Dindal, D.L. (Ed.), Soil Biology as Related to Land Use Practices. Proceedings of the $7^{\text {th }}$ International Soil Colloquium, Washington, D.C., pp. 759-769.

Wallwork, J.A., MacQuitty, M., Silva, S. \& Whitford, W.G. (1986) Seasonality of some Chihuahuan desert soil oribatid mites (Acari: Cryptostigmata). Journal of Zoology, A, 208 (3), 403-416. https://doi.org/10.1111/j.1469-7998.1986.tb01903.x

Walter, D.E. \& Proctor, H.C. (2013) Mites. Ecology, Evolution and Behaviour. Life at a Microscale. Springer, $2^{\text {nd }}$ edition, 494 pp. https://doi.org/10.1007/978-94-007-7164-2

Webb, N.R. (1977) Observations on Steganacarus magnus, general biology and life cycle. Acarologia, 19 (4), $686-696$.

Webb, N.R. (1989) Observations on the life cycle of Steganacarus magnus (Acari: Cryptostigmata). Pedobiologia, 33, 293299.

Weigmann, G. (1975) Labor- und Freilanduntersuchungen zur Generationsdauer von Oribatiden (Acari: Oribatei). Pedobiologia, $15,133-148$.

West, C. (1982) Life histories of three species of sub-antarctic oribatid mite. Pedobiologia, 23, 59-67.

Woodring, J.P. (1965) The biology of five new species of Oribatids from Lousiana. Acarologia, 7 (3), 564-576.

Woodring, J.P. \& Cook, E.F. (1962) The biology of Ceratozetes cisalpinus Berlese, Scheloribates laevigatus Koch, and Oppia neerlandica Oudemans (Oribatei), with a description of all stages. Acarologia, 4, 101-137.

Wunderle, I. (1991) Life-histories and notes on the behavior of tree-living oribatid mites. In: Dusbábek, F. \& Bukva, V. (Eds.), Modern Acarology, Vol. 2. Prague and SPB Academic Publishing, The Hague, Prague, pp. 529-535. 